Pacific Journal of Mathematics

ON RADICALS AND PRODUCTS

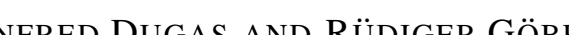




\title{
ON RADICALS AND PRODUCTS
}

\section{MANFREd DUgaS AND RÜDIGER GöBEL}

\begin{abstract}
An Abelian group $G$ is called cotorsion-free if 0 is the only pure-injective subgroup contained in $G$. If $G$ is a cotorsion-free Abelian group, we construct a slender, $\boldsymbol{\aleph}_{1}$-free Abelian group $A$ such that $\operatorname{Hom}(A, G)=$ 0 . This will be used to answer some questions about radicals and torsion theories of Abelian groups.
\end{abstract}

0. Introduction. In this paper we will consider torsion free abelian groups from I. Kaplansky's point of view: "In this strange part of the subject anything that can conceivably happen actually does happen", cf. [K, p. 81]. This statement which is supported by classical results holds in an even more spectacular sense which was not expected at this time. There are many results on torsion free abelian groups which are undecidable in ZFC, the axioms of Zermelo-Fränkel set theory including the axiom of choice. The first surprising result of this kind after years of stagnation was Shelah's solution of the famous Whitehead problem [S1]. In this paper Shelah also constructed for the first time arbitrarily large indecomposable abelian groups, thus improving classical results of S. Pontrjagin, R. Baer, I. Kaplansky, L. Fuchs, A. L. S. Corner and others, compare [Fu2, Vol. II] and $[\mathbf{K}]$. Indecomposable abelian groups are necessarily cotorsion-free with only a few exceptions. These are the cyclic groups of prime power $Z_{p^{n}}$, the Prüfer groups $Z\left(p^{\infty}\right)$, the group of rational numbers $\mathbf{Q}$ and the additive group $J_{p}$ of $p$-adic integers. A group is called cotorsion-free if and only if it contains only the trivial cotorsion subgroup 0, cf. [GW1]. Remember that $C$ is cotorsion (in the sense of $\mathrm{K}$. H. Harrison) if $\operatorname{Ext}_{\mathbf{z}}(\mathbf{Q}, C)=0$. From simple properties of cotorsion groups we conclude that a group $G$ is cotorsion-free if and only if $G$ is torsion-free $\left(Z_{p} \nsubseteq G\right)$, reduced $(\mathbf{Q} \nsubseteq G)$ and $J_{p} \nsubseteq G$ for all primes $p$, cf. [GW1]. For countable groups cotorsion-free is the same as reduced and torsion-free. A. L. S. Corner's celebrated theorem indicates then that each ring with a countable and cotorsion-free additive structure is the endomorphism ring of some (cotorsion-free) abelian group, cf. [Fu1, Vol. II]. This result was extended by the authors [DG2] to arbitrary rings with cotorsion-free additive groups which are then realized on arbitrarily large cotorsion-free abelian groups. Using rings without non-trivial idempotents, indecomposable groups of 
any size can be obtained and the aforementioned result becomes a trivial consequence of [DG2]. However, using other elementary ring constructions this result supports Kaplansky's point of view in many aspects, e.g. there are many new different counter examples for I. Kaplansky's test problems. Similar results which are in many cases even undecidable im ZFC have been derived in [DG1], [EM], [Me], [DH1] and others.

One of the questions "close" to results undecidable in $\mathrm{ZFC}$ is related with "rigid systems". A class $\left\{A_{i}, i \in I\right\}$ of abelian groups is semi-rigid if $\operatorname{Hom}\left(A_{i}, A_{j}\right) \neq 0 \neq \operatorname{Hom}\left(A_{j}, A_{i}\right)$ implies $i=j$ for any $i, j \in I$. This class is rigid if already $\operatorname{Hom}\left(A_{i}, A_{j}\right) \neq 0$ implies $i=j$. The class is proper if $I$ is not a set. M. Dugas and S. Herden [DH1] constructed proper rigid classes of (indecomposable) abelian groups using Gödel's axiom of constructibility $V=L$. Such a result cannot be expected in ZFC alone as follows from the Vopenka principle. However, at least semi-rigid proper classes exist in ZFC as recently shown by R. Göbel and S. Shelah [GS]. This result is based on a construction of arbitrarily large cotorsion-free abelian groups $A$ with the property that $U=A$ for any subgroup $U \subseteq A$ with $|U|=|A|$ and $A / U$ cotorsion free.

All these constructions are highly sophisticated using transfinite induction on generating elements. The very heart of this paper is a similar kind of result based on a much simpler construction. Due to the elementary construction of the groups (4.2) we are able to pose stronger conditions on their structure, which allow us to answer some open problems and give new solutions to some already settled problems. These extra conditions are the properties $\aleph_{1}$-free and slender. A group is called $\boldsymbol{\aleph}_{1}$-free if all its countable subgroups are free. The most popular non-free $\aleph_{1}$-free groups are products $\mathbf{Z}^{*}$ of the integers, in particular the Baer-Specker group $\mathbf{Z}^{\aleph_{0}}$. The proof that $\mathbf{Z}^{\aleph_{0}}$ is $\boldsymbol{\aleph}_{1}$-free and not free is due to R. Baer and E. Specker, cf. [Fu1, Vol. I]. We will use R. J. Nunke's well-known characterization of slender groups as a definition. Hence a group is slender if and only if it is cotorsion free and if it does not contain a copy of the Baer-Specker group. Then we have the following quite powerful

THEOREM. If $G$ is a cotorsion-free abelian group and $\lambda$ a strong limit cardinal of cofinality $\omega$ with $|G| \leq \lambda$, then we find an $\aleph_{1}$-free and slender abelian group $A$ of size $|A|=2^{\lambda}$ such that $\operatorname{Hom}_{\mathbf{Z}}(A, G)=0$.

There is a proper class of the required cardinals $\lambda$ and hence we have a proper class of slender and $\boldsymbol{\aleph}_{1}$-free groups $A$ with $\operatorname{Hom}(A, G)=0$. Moreover there exist a proper semi-rigid class of $\boldsymbol{\aleph}_{1}$-free and slender groups. 
First we obtain a new and totally different solution for Problem $78 b$ in L. Fuchs [Fu1, Vol. II, p. 184]. This problem was already solved in [GW2] and the answer is as follows. There does not exist a set of abelian groups such that all slender groups can be obtained by constructing inductively extensions, direct sums and subgroups. Second we obtain a new solution for a problem stated in [GW2]. This problem was originally solved in [GS] and the answer is as follows. The class of cotorsion-free abelian groups is not singly cogenerated as a torsion theory. This means that it is not possible to obtain all cotorsion-free groups by constructing inductively extensions, cartesian products and subgroups from a given set of groups.

Besides these new proofs of older results we simultaneously obtain answers to some open problems which are generally known and may be found in [FOW 1, 2] for instance. These problems are related to torsion theories and radicals. A pair $(\mathscr{T}, \mathscr{F})$ of classes of abelian groups is a torsion theory if the classes $\mathscr{T}$ and $\mathscr{F}$ satisfy "similar" closure properties as the pair (torsion abelian groups, torsion free abelian groups). A precise definition is given in the books [St] or [L] and at the beginning of $\S 4$. The main result extends [DH1] and [GS]. It says that in a model of ZFC without measurable cardinals [i.e. ZFC $+\nexists \boldsymbol{\aleph}_{m}$ (= first measurable cardinal)] the torsion theory ( $T \mathscr{P}$, strongly cotorsion-free) is neither singly generated nor singly cogenerated. The existence of such torsion theories in $\mathrm{ZFC}+\not \nexists \aleph_{m}$ was unknown. By $T \mathscr{P}$ we denote the torsion class generated by $\mathscr{P}=\left\{\mathbf{Z}^{\kappa} / \mathbf{Z}^{<\kappa}, \kappa\right.$ any regular cardinal $\}$ compare $\$ 1$. Strongly cotorsion-free groups are special cotorsion-free groups and may be defined as the torsion-free class associated with $T \mathscr{P}$, cf. $\S 4$.

Torsion theories naturally lead to radicals. They are considered first in §2. Our main result is (2.4). We will show that radicals related to strongly cotorsion-free groups commute with products of size less than $\aleph_{m}$. This does not hold for the more general class of cotorsion-free groups and the radical $R_{\mathbb{Z}}$ related to the integers does not commute with products of size $\aleph_{m}$. Here we will use results of J. Los and answer a question which goes back to B. Charles, compare also [Fu1, Vol. I, p. 71, Problem 7].

In $\S 5$ we will investigate the Chase radical $\nu_{1} A=\cap\{U \subseteq A, A / U$ is $\aleph_{1}$-free $\}$ of an abelian group $A$. The Chase radical has a related torsion theory ( $\left\{A, \nu_{1} A=A\right\}, \boldsymbol{\aleph}_{1}$-free). This is singly generated (5.1) but not singly cogenerated. This was another open problem answered by the Theorem above. Finally we will compute some radicals explicitly in $\S 6$.

1. Definitions and notations. $\mathrm{ZFC}=$ Zermelo-Fränkel set theory and axiom of choice. $\kappa$ will always denote a cardinal and $\kappa^{+}$its successor 
cardinal. Some special cardinals are

$\boldsymbol{\aleph}_{0}=\omega=$ first infinite cardinal,

$|X|=$ cardinality of a set $X$,

$\aleph_{m}=$ first measurable cardinal.

The axiom " $O \#$ exists" can be found in T. Jech [ $\mathbf{J}$, p. 339]. It is equivalent to each of the following two statements (Kunen, cf. [J, p. 339]).

(1) For a limit ordinal $\lambda$, the model $\left\langle L_{\lambda}, P\right\rangle$ has an uncountable set of indiscernibles.

(2) There exists a nontrivial elementary embedding of $L$ into $L$.

Let $\left\{A_{i}, i \in \kappa\right\}$ be a family of abelian groups. We let $\Pi_{i \in \kappa} A_{i}=$ (cartesian) product. If $f \in \prod_{i \in \kappa} A_{i}$, then $[f]=\left\{i \in \kappa, f(i)=f_{i} \neq 0\right\}$ is the support of $f . \prod_{i \in \kappa} A_{i}=A^{\kappa}$ if $A_{i}=A$ for all $i \in \kappa$. Some special subgroups are

$$
\prod_{i \in \kappa}^{<\kappa} A_{i}=\left\{f \in \prod_{i \in \kappa} A_{i},|[f]|<\kappa\right\}
$$

and in particular

$$
A^{<\kappa}=\left\{f \in A^{\kappa},|[f]|<\kappa\right\}, \quad A^{(\kappa)}=\left\{f \in A^{\kappa},|[f]|<\aleph_{0}\right\} .
$$

Let $A_{\kappa}=A^{\kappa} / A^{<\kappa}$ and in particular $\mathbf{Z}_{\kappa}=\mathbf{Z}^{\kappa} / \mathbf{Z}^{<\kappa}$.

Let $F$ be a subfunctor of the identity if $F A \subseteq A$ for all abelian groups $A$ such that if $\alpha: A \rightarrow B$ is a homomorphism, then $(F A)^{\alpha} \subseteq F B$. If $\left\{U_{i} \subseteq A_{i}, i \in \kappa\right\}$, we identify $\prod_{i \in \kappa} U_{i} \subseteq \prod_{i \in \kappa} A_{i}$ in the natural way. We say that $F$ commutes with $\prod_{\kappa}$ (with products of size $\left.\leq \kappa\right)$ if $F \prod_{i \in \kappa} A_{l}=$ $\prod_{i \in \kappa} F A_{i}$ for all families $\left\{A_{i}, i \in \kappa\right\}$ of abelian groups.

Let $x \mid y$ denote that $x$ divides $y$.

If $f: X \rightarrow Y$ and $T \subseteq X$, then $f \uparrow X=\left.f\right|_{X}$ denotes the restriction map.

2. Singly generated radicals. Let $\mathscr{X}$ be a class of groups. With every abelian group $A$ we associate a subgroup $R_{\mathscr{X}} A=\bigcap_{\varphi: A \rightarrow X \in \mathscr{X}} \operatorname{ker} \varphi$. This is a well-known construction and $R_{\mathscr{X}}$ is a subfunctor of the identity compare L. Fuchs [Fu1, Vol. I, §6] and §1. Using some terminology of Philip Hall this functor is $R_{\mathscr{X}}=\mathscr{X}^{-Q}$ and the groups on which $R_{\mathscr{X}}$ operates as the identity are called $\mathscr{X}$-perfect, compare D. J. S. Robinson [Ro, part 2], R. Baer [B, p. 287] and [G1]. If $\mathscr{X}=\{X\}$ is a singleton, we write $R_{\mathscr{X}}=R_{X}$ and $R_{X}$ is then called a singly generated radical compare T. H. Fay, E. P. Oxford and G. L. Walls [FOW2]. Observe that $R_{\mathscr{X}}$ is always a radical, i.e. $R_{\mathscr{X}}\left(A / R_{\mathscr{X}} A\right)=0$. 
Since $R_{\mathscr{X}}$ is a subfunctor of the identity, $R_{\mathscr{X}}$ commutes with direct sums, i.e. $R_{\mathscr{X}}\left(\oplus_{i \in I} A_{i}\right)=\bigoplus_{i \in I} R_{\mathscr{X}} A_{i}$; compare L. Fuchs [Fu1, Vol. I, p. 29, Exercis 9]. Therefore the natural question arises under which conditions $R_{\mathscr{X}}$ commutes with direct products. This is a special case of Problem 7 in the book of L. Fuchs [Fu1, Vol. I, p. 71]. [FOW1] show that $R_{X}$ commutes with countable products if and only if $X$ is stout in the sense of [G1]. This is equivalent to saying that $X$ is cotorsion-free. Recall that $X$ is cotorsion-free iff $X$ has only the trivial cotorsion subgroup 0 or equivalently $X$ is reduced, torsion-free and has no subgroup isomorphic to the $p$-adic integers $J_{p}$, compare R. Göbel [G1, p. 41 and p. 49, Theorem 4.1], R. Göbel and B. Wald [GW1, p. 210, Folgerung 4.2]. Further equivalent conditions may be found in M. Dugas and R. Göbel [DG1]. In the same paper [FOW1] say that it is an open problem whether $R_{X}$ commutes with arbitrary products. In order to answer this question we will use the following

DEFinition 2.1. A group $X$ is strongly cotorsion-free if and only if $\operatorname{Hom}\left(\mathbf{Z}_{\kappa}, X\right)=0$ for all regular cardinals $\kappa \geq \aleph_{1}$ with $\kappa<\aleph_{m}$. Recall our notation $\mathbf{Z}_{\kappa}=\mathbf{Z}^{\kappa} / \mathbf{Z}^{<\kappa}$.

A simple application of a well-known result of S. Balcerzyk shows that $\operatorname{Hom}\left(\mathbf{Z}_{\omega}, X\right)=0$ if and only if $X$ is cotorsion-free, compare R. Göbel and B. Wald [GW1, p. 213, Satz 4.6]. We will see in (2.3) that strongly cotorsion-free implies cotorsion-free. Strongly cotorsion-free is the same class as all groups which are $\mu / \kappa$-reduced for all cardinals $\mu \in \kappa$ in B. Wald [W2]. We have the immediate observation that every countable cotorsion-free group is slender. It follows from B. Wald [W2, Theorem $3.4]$, that cotorsion-free groups of cardinality $\aleph_{1}$ are also strongly cotorsion-free in $V=L$.

Sometimes we use the following homomorphism $\tilde{\mu}: \mathbf{Z}^{\aleph_{m}} \rightarrow \mathbf{Z}$ which is induced from a $\sigma$-additive measure $\mu: \mathscr{P}\left(\boldsymbol{\aleph}_{m}\right) \rightarrow\{0,1\}$. If $f=\left(f_{i}\right)_{i \in \aleph_{m}} \in$ $\mathbf{Z}^{\aleph_{m}}$ and $z^{-1}=\left\{i \in \boldsymbol{\aleph}_{m}, f_{i}=z\right\}$ for $z \in \mathbf{Z}$, then $\boldsymbol{\aleph}_{m}=\dot{U}_{z \in \mathbf{Z}} z^{-1}$. Hence there is precisely one $z \in \mathbf{Z}$ such that $\mu\left(z^{-1}\right)=1$. Therefore

$$
\tilde{\mu}(f)=z \quad \text { iff } \mu\left(z^{-1}\right)=1
$$

defines a homomorphism from $\mathbf{Z}^{\boldsymbol{\aleph}_{m}}$ onto $\mathbf{Z}$.

Since $\boldsymbol{\aleph}_{m}$ is measurable we have $\tilde{\mu}\left(\mathbf{Z}^{<\boldsymbol{\kappa}_{m}}\right)=0$ and $\tilde{\mu}$ induces an epimorphism from $\mathbf{Z}_{\aleph_{m}}$ to $\mathbf{Z}$. Hence we see that $\operatorname{Hom}\left(\mathbf{Z}_{\aleph_{m}}, X\right)=0$ implies $X=0$. This explains our assumption $\kappa<\aleph_{m}$ in (2.1).

Proposition 2.3.

(a) Strongly cotorsion-free implies cotorsion-free, 
(b) residually slender implies strongly cotorsion-free,

(c) slender implies residually slender.

Proof. (a) If $0 \neq C \subseteq X$ is cotorsion and $X$ is strongly cotorsion-free then we may assume w.l.o.g. that $C$ is algebraically compact. Take any pure and countable subgroup $A$ of $\mathbf{Z}_{\kappa}$ (which always exists). Since $\kappa$ is regular and $\kappa \geq \boldsymbol{\aleph}_{1}$, also $\mathbf{Z}_{\kappa}$ is $\boldsymbol{\aleph}_{1}$-free as follows from B. Franzen [Fr] or B. Wald [W1]. Therefore $A$ is free. We remark that this can also be derived from the Wald-Los-Lemma 2.6 which implies $A \subseteq \mathbf{Z}^{\kappa}$. Then $A$ is free by a result of Baer and Specker, cf. L. Fuchs [Fu1, Vol. I, p. 94, Theorem 19.2]. Therefore we find a non-trivial homomorphism from $A$ into $C$. Since $A$ is a pure subgroup of $\mathbf{Z}_{\kappa}$ and $C$ is pure injective, this homomorphism extends to a non-trivial homomorphism from $\mathbf{Z}_{\kappa}$ into $X$ which is excluded by Definition 2.1 .

(b) and (c). Here we only have to recall a theorem of J. Loš and the definition residually- $\mathscr{X}$ : A group $X$ has this property if

$$
\bigcap\{U \subseteq X, X / U \in \mathscr{X}\}=0 .
$$

This is equivalent to say that $X$ is a subgroup of a product of $\mathscr{X}$-groups. If $X$ is residually slender and $0 \neq \sigma \in \operatorname{Hom}\left(\mathbf{Z}_{\kappa}, X\right)$ for some cardinal $\kappa$, then we find a homomorphism $\pi \in \operatorname{Hom}(X, S)$ for some slender group $S$ such that $\sigma \pi \neq 0$. From the theorem of Loš we obtain that $\kappa \geq \boldsymbol{\aleph}_{m}$, compare L. Fuchs [Fu1, Vol. II, p. 161, Theorem 94.4(ii)]. Therefore $X$ is strongly cotorsion free.

THEOREM 2.4 .

(1) If $\mathscr{X}$ is a class of strongly cotorsion-free abelian groups, then $R_{\mathscr{X}}$ commutes with $\Pi_{\kappa}$ for all cardinals $\kappa<\boldsymbol{\aleph}_{m}$.

(2) If $\kappa$ is a regular cardinal, $\kappa \geq \boldsymbol{\aleph}_{0}$, there is a cotorsion-free group $G=G(\kappa)$ of size $|G|=2^{\kappa^{+}}$such that $R_{G}$ does not commute with $\Pi_{\kappa^{+}}$. More precisely there are groups $G_{\alpha}\left(\alpha \in \kappa^{+}\right)$with $R_{G} G_{\alpha}=G_{\alpha}$ for all $\alpha \in \kappa^{+}$and $R_{G} \Pi_{\alpha \in \kappa^{+}} G_{\alpha} \neq \Pi_{\alpha \in \kappa^{+}} G_{\alpha}$.

From (2.3) and (2.4) we have an immediate

COROLlARY 2.5. If $\mathscr{X}$ is a class of residually slender groups, then $R_{\mathscr{X}}$ commutes with $\prod_{\kappa}$ for all cardinals $\kappa<\aleph_{m}$.

REMARK. (2.4)(1) and (2.5) improve Theorem 3.2 in [FOW2] which is the case $\kappa=\boldsymbol{\aleph}_{0}$. Part of $(2.5)$ is contained in [Ga]. (2.4)(2) answers an open problem, mentioned in [FOW2]. 
Proof of (2.4).

(1) will be proved by contradiction. Assume that $\kappa$ is a minimal cardinal such that (1) does not hold.

First we will show that $\kappa$ must be regular and $\kappa \geq \boldsymbol{\aleph}_{1}$. If $\kappa$ is not regular, then $\lambda=c f(\kappa)<\kappa$. Hence $\kappa=\bigcup_{\alpha \in \lambda} I_{\alpha}$ for some sets $I_{\alpha}$ of cardinality $\left|I_{\alpha}\right|<\kappa$. We conclude

$$
R_{\mathscr{X}}\left(\prod_{i \in \kappa} A_{i}\right)=R_{\mathscr{X}}\left(\prod_{\alpha \in \lambda} \prod_{i \in I_{\alpha}} A_{i}\right)=\prod_{\alpha \in \lambda} \prod_{i \in I_{\alpha}} R_{\mathscr{X}} A_{i}=\prod_{i \in \kappa} R_{\mathscr{X}} A_{i}
$$

from the minimality of $\kappa$. Therefore $\kappa$ must be regular. If $\kappa=\boldsymbol{\aleph}_{0}$, then $R_{X}$ commutes with $\Pi_{\aleph_{0}}$ for all cotorsion-free groups $X$ as shown in [FOW2, Theorem 3.2]. Therefore

$$
\begin{aligned}
R_{\mathscr{X}}\left(\prod_{i \in \aleph_{0}} A_{i}\right) & =\bigcap_{X \in \mathscr{X}} R_{X} \prod_{i \in \aleph_{0}} A_{i}=\bigcap_{X \in \mathscr{X}} \prod_{i \in \aleph_{0}} R_{X} A_{i} \\
& =\prod_{i \in \aleph_{0}}\left(\bigcap_{X \in \mathscr{X}} R_{X} A_{i}\right)=\prod_{i \in \aleph_{0}} R_{\mathscr{X}} A_{i}
\end{aligned}
$$

and (1) is shown for $\kappa=\boldsymbol{\aleph}_{0}$. Hence $\kappa \geq \boldsymbol{\aleph}_{1}$.

Since $\kappa$ is a minimal counterexample, we find groups $A_{i}$ such that $\prod_{i \in \kappa} R_{\mathscr{X}} A_{i} \neq R_{\mathscr{X}} \prod_{i \in \kappa} A_{i}$. However $R_{\mathscr{X}} \prod_{i \in \kappa} A_{i} \subseteq \Pi_{i \in \kappa} R_{\mathscr{X}} A_{i}$ holds trivially and so we find $a \in \prod_{i \in \kappa} R_{\mathscr{X}} A_{i} \backslash R_{\mathscr{X}} \prod_{i \in \kappa} A_{i}$. By definition of $R_{\mathscr{X}} \prod_{i \in \kappa} A_{i}$, there is a homomorphism $\varphi: \prod_{i \in \kappa} A_{i} \rightarrow X$ for some $X \in \mathscr{X}$ such that $\varphi(a) \neq 0$. If $x \in \prod_{i \in \kappa}^{<\kappa} R_{\mathscr{X}} A_{i}$ then $x \in \Pi_{i \in \lambda} R_{\mathscr{X}} A_{i}$ for some $\lambda<\kappa$. From the minimality of $\kappa$ we have $R_{\mathscr{X}} \prod_{i \in \lambda} A_{i}=\prod_{i \in \lambda} R_{\mathscr{X}} A_{i}$ and we conclude $\varphi(x)=0$. Therefore $\varphi(K)=0$ where $K=\prod_{i \in \kappa}^{<\kappa} R_{\mathscr{X}} A_{i}$ and $\varphi$ induces a homomorphism $\varphi: \prod_{i \in \kappa} R_{\mathscr{X}} A_{i} / K \rightarrow X$ with $(a+K)^{\varphi} \neq 0$. Define $\sigma: \mathbf{Z}^{\kappa} \rightarrow \prod_{i \in \kappa} R_{\mathscr{X}} A_{i}$ by $\left(z_{i}\right)_{i \in \kappa}^{\sigma}=\left(a_{i} z_{i}\right)_{i \in \kappa}$ where $a=\left(a_{i}\right)_{i \in \kappa}$. Then $\sigma \varphi: \quad \mathbf{Z}^{\kappa} \rightarrow X$ and $(1)_{i \in \kappa}^{\sigma \varphi}=(a+K)^{\varphi} \neq 0$. Since $K^{\varphi}=0$, also $\left(\mathbf{Z}^{<\kappa}\right)^{\sigma \varphi}=0$. Hence $\sigma \varphi$ induces a non-trivial homomorphism from $\mathbf{Z}_{\kappa}$ into $X$. This contradicts that $X$ is strongly cotorsion-free.

(2) If $\kappa$ is regular or $\kappa=\aleph_{0}$, we choose an orthogonal system $\left\{G_{\alpha}\right.$, $\left.\alpha \in \kappa^{+}\right\}$of groups such that $\left|G_{\alpha}\right|=\kappa$. By orthogonal we understand $\operatorname{Hom}\left(G_{\alpha}, G_{\beta}\right)=0$ for all different $\alpha, \beta \in \kappa$. If $\kappa=\boldsymbol{\aleph}_{0}$ such a system was constructed by R. Baer, using rank-1 groups, compare L. Fuchs [Fu1, Vol. II, p. 110]. Systems for cardinals $\kappa$ less than the first strongly inaccessible cardinal are due to A. L. S. Corner and L. Fuchs, compare L. Fuchs [Fu1, Vol. II, p. 130, Theorem 89.2]. Later L. Fuchs [Fu2] constructed orthogonal systems for $\kappa \leq \aleph_{m}$ and independently S. Shelah [S1] proved the existence of such systems for all regular cardinals $\kappa$.

Now choose $G=G(\kappa)=\prod_{\alpha \in \kappa^{+}} G_{\alpha} / \Pi_{\alpha \in \kappa^{+}}^{<\kappa^{+}} G_{\alpha}$ and let $\pi$ denote the canonical epimorphism from $\prod_{\alpha \in \kappa^{+}} G_{\alpha}$ onto $G$. Then we will use the Wald-Loš-Lemma which is shown below. 
If $\varphi: G_{\beta} \rightarrow G$ is any homomorphism, there exists $\gamma: G_{\beta} \rightarrow \prod_{\alpha \in \kappa^{+}} G_{\alpha}$ such that $\gamma \pi=\varphi$. Since $\left\{G_{\alpha}, \alpha \in \kappa^{+}\right\}$is orthogonal, we have $G_{\beta}^{\gamma} \subseteq G_{\beta} \subseteq$ $\Pi_{\alpha \in \kappa^{+}}^{<\kappa} G_{\alpha}$ and therefore $\varphi=\gamma \pi=0$. We conclude $R_{G} G_{\alpha}=G_{\alpha}$ for all $\alpha \in \kappa$ from the definition of $R_{G}$. On the other hand, by definition of $G$ we have

$$
R_{G}\left(\prod_{\alpha \in \kappa^{+}} G_{\alpha}\right) \subseteq \prod_{\alpha \in \kappa^{+}}{ }^{<\kappa} G_{\alpha} .
$$

We combine these results and conclude

$$
R_{G}\left(\prod_{\alpha \in \kappa^{+}} G_{\alpha}\right) \subseteq \prod_{\alpha \in \kappa^{+}}{ }^{<\kappa} G_{\alpha} \subset \prod_{\alpha \in \kappa^{+}} G_{\alpha}=\prod_{\alpha \in \kappa^{+}} R_{G} G_{\alpha}
$$

and (2) is shown.

The following lemma was used in (2.4)(2). A special case was first shown by $\mathbf{J}$. Los [L] and the idea of its proof is also contained (implicitly) in G. A. Reid [R, p. 27]. It is stated in a quite general form by B. Wald [W1] which uses complete filters. His elegant proof extends trivially. Since it is also very short, we will include it as

WALD-LoŠ-LEMMA 2.6. Let $\left\{G_{\alpha}, \alpha \in \kappa\right\}$ be a family of groups and $\phi$ a $\kappa$-complete filter on $\kappa$, i.e. $\phi$ is a filter on $\kappa$ and intersections of less than $\kappa$ elements of $\phi$ belong to $\phi$. If $\kappa$ is regular and $A \stackrel{\sigma}{\rightarrow} \prod_{\alpha \in \kappa} G_{\alpha} / \phi$ is an embedding with $|A|<\kappa$, then there is an induced monomorphism $\gamma: A \rightarrow$ $\Pi_{\alpha \in \kappa} G_{\alpha}$ with $\gamma \pi=\alpha$ for the canonical map $\pi: \prod_{\alpha \in \kappa} G_{\alpha} \rightarrow \prod_{\alpha \in \kappa} G_{\alpha} / \phi$.

REMARK. If $U=\left\{g \in \Pi_{\alpha \in \kappa} G_{\alpha}, \kappa \backslash[g] \in \phi\right\}$, then $\Pi_{\alpha \in \kappa} G_{\alpha} / \phi=$ $\prod_{\alpha \in \kappa} G_{\alpha} / U$. The particular filter $\{I \subseteq \kappa,|\kappa \backslash I|<\kappa\}$ is used in (2.4)(2).

Proof (cf. B. Wald [W1]). Let $\pi: \prod_{\alpha \in \kappa} G_{\alpha} \rightarrow \Pi_{\alpha \in \kappa} G_{\alpha} / \phi=G$ be the canonical projection $\sigma=\mathrm{id}$ and $\left\{\bar{a} \in \prod_{\alpha \in \kappa} G_{\alpha}, a \in A\right\}$ a set of representatives of $A$ in $\prod_{\alpha \in \kappa} G_{\alpha}$ such that $\bar{a}^{\pi}=a$ for all $a \in A$.

For $g \in G$, let $O(g)=\{\alpha<\kappa, g(\alpha)=0\}$.

If $a, b \in A$, then $a+b-(a+b)=0$ and therefore

$$
O(\bar{a}+\bar{b}-(\overline{a+b})) \in \phi .
$$

Let $D=\bigcap_{a, b \in A} O(\bar{a}+\bar{b}-(\overline{a+b}))$, which is an element of $\phi$ as follows from $|A|<\kappa$. We denote by $a^{\gamma}=\left.\bar{a}\right|_{D}$ the restriction of $\bar{a}$ to $D$. Then $\gamma: A \rightarrow \prod_{\alpha \in \kappa} G_{\alpha}$ is a monomorphism with $\gamma \pi=\mathrm{id}_{A}$.

The next theorem will show that the requirement $\kappa<\boldsymbol{\aleph}_{m}$ in (2.4)(1) is also necessary. 


\section{THEOREM 2.7. $R_{\mathrm{Z}}$ does not commute with $\Pi_{\aleph_{m}}$.}

Proof ${ }^{1}$. Let $\mu$ be a $\{0,1\}$-measure on $\aleph_{m}$. Then $\mu$ may be replaced by a normal measure on $\boldsymbol{\aleph}_{m}$ which we also denote by $\mu$; apply $\mathrm{T}$. Jech [J, p. 317, Lemma 28.11]. Recall that $\mu$ is normal on $\aleph_{m}$ if the following holds:

If $f: \boldsymbol{\aleph}_{m} \rightarrow \boldsymbol{\aleph}_{m}$ is any regressive function (i.e. $f(\alpha)<\alpha$ for all $\left.\alpha \in \aleph_{m} \backslash 0\right)$ and if $S \subseteq \aleph_{m}$ with $\mu S=1$, then there exists a set $T \subseteq S$ such that $\mu T=1$ and $f \uparrow T$ is constant; cf. T. Jech [J, p. 316].

Since $\mu$ is normal, we derive

$$
\mu\left(\left\{\alpha \in \aleph_{m}, \alpha \text { regular cardinal }\right\}\right)=1 ;
$$

cf. T. Jech [J, p. 317, Lemma 28.12]. Let $\bar{\alpha}$ be order isomorphic to $\alpha$ for all $\alpha \in \aleph_{m}$ and construct the disjoint union $I=\dot{U}_{\alpha \in S} \bar{\alpha}$ with $S$ the set of all regular cardinals $\left\langle\boldsymbol{\aleph}_{m}\right.$. Assume $\alpha \in S$ in the following. Each subset $X \subseteq I$ will be associated with a set $F(X) \subseteq \aleph_{m}$ if we let $\beta \in F(X)$ if and only if $\mu(\{\alpha, \beta \in X \cap \bar{\alpha}\})=1$. We define $X \in U$ if and only if $\mu F(X)=$ 1. This is a kind of product measure. Now we want to show that $U$ is an ultrafilter on $I$. If $X \notin U$ then $\mu F(X)=0$ and $F(X)^{c}=F\left(X^{c}\right)$ implies $\mu F\left(X^{c}\right)=1$. Since $U$ is obviously a filter it is also an ultrafilter. To see that $U$ is $\boldsymbol{\aleph}_{m}$-complete, we assume $\kappa<\boldsymbol{\aleph}_{m}$ and $X_{i} \in U$ for $i \in \kappa$.

If $\beta \in \bigcap_{i<\kappa} F\left(X_{i}\right)$ then for all $i<\kappa, \mu\left(\left\{\alpha, \beta \in X_{i} \cap \bar{\alpha}\right\}\right)=1$. Hence

$$
\begin{aligned}
1 & =\mu\left(\bigcap_{i<\kappa}\left\{\alpha, \beta \in X_{i} \cap \bar{\alpha}\right\}\right)=\mu\left(\left\{\alpha, \beta \in \bigcap_{i<\kappa} X_{i} \cap \bar{\alpha}\right\}\right) \\
& =\mu\left(F\left(\bigcap_{i<\kappa} X_{i}\right)\right)
\end{aligned}
$$

and $\bigcap_{i<\kappa} X_{i} \in U$. We want to show that the $\aleph_{m}$-complete ultrafilter $U$ extends the following $\boldsymbol{\aleph}_{m}$-complete filter

$$
\mathscr{F}=\{X \subseteq I, \mu(\{\alpha \in S,|\bar{\alpha} \backslash X|<|\alpha|\})=1\} .
$$

We will show that $X \in \mathscr{F}$ implies $X \in U$.

Define a function $f: \aleph_{m} \rightarrow \boldsymbol{\aleph}_{m}$ setting $f(\alpha)=\sup (\bar{\alpha} \backslash X)$ if $\alpha \in S$ and $f(\alpha)=0$ if $\alpha \in \boldsymbol{\aleph}_{m} \backslash S$. Since $f(\alpha)<\alpha, f$ is a regressive function. From $X \in \mathscr{F}$ we have $\mu(\{\alpha \in S,|\bar{\alpha} \backslash X|<|\alpha|\})=1$ and since $f$ is regressive and $\mu$ is normal we also find $T \subseteq\{\alpha \in S,|\bar{\alpha} \backslash X|<|\alpha|\}$ with measure 1 and $f \mid T=$ const. $=\beta \in \boldsymbol{\aleph}_{m}$. If we pick any $\gamma \in \aleph_{m} \backslash \beta$ then obviously $\{\alpha \in S$, $\gamma \in \bar{\alpha} \backslash X\} \supseteq T \cap\{\alpha \in S, \bar{\alpha}>\gamma\}$. However

$$
\begin{aligned}
1 & \geq \mu(\{\alpha, \gamma \in \bar{\alpha} \backslash X\}) \geq \mu(T \cap\{\alpha, \bar{\alpha}>\gamma\}) \\
& \geq \min [\mu T,(\{\alpha, \bar{\alpha}>\gamma\})]=1 .
\end{aligned}
$$

\footnotetext{
${ }^{1}$ We would like to thank Alan Mekler for explaining to us how the normal measure can be used to show the Theorem. Due to this we are able to present the stronger form (2.7) of our original result.
} 
We obtain $\mu(\{\alpha, \gamma \in \bar{\alpha} \backslash X\})=1$ and by definition of $F$ also $\gamma \in F(X)$. Therefore $F(X) \supseteq\left(\boldsymbol{\aleph}_{m} \backslash \beta\right)$ are sets of measure 1. So $X \in U$ follows from $F(X)=1$.

Let $\rho$ be the $\boldsymbol{\aleph}_{m}$-additive measure induced by $U$. Hence we have the epimorphism

$$
\tilde{\rho}: \mathbf{Z}^{I}=\prod_{\alpha \in S} \mathbf{Z}^{\alpha} \rightarrow \mathbf{Z} \quad \text { from (2.2). }
$$

Remember that $|\alpha|$ is a regular cardinal. Let $\prod_{\alpha \in S} \mathbf{Z}^{<|\alpha|}$ where $\mathbf{Z}^{<|\alpha|}$ is the set of all maps $f: \bar{\alpha} \rightarrow \mathbf{Z}$ with support of cardinality $<|\alpha|$. If $f \in \Pi_{\alpha \in S} \mathbf{Z}^{<|\alpha|}$ and $O(f)=\{i \in I, f(i)=0\}$, then $|\bar{\alpha} \backslash O(f)|<|\alpha|$ and therefore $O(f)$ $\in \mathscr{F} \subseteq U$.

By definition of $\tilde{\rho}$ and $\rho(O(f))=1$ we obtain $\tilde{\rho}(f)=0$. Therefore $\tilde{\rho}$ induces an epimorphism

$$
\hat{\rho}: \prod_{\alpha \in S} \mathbf{Z}^{\alpha} / \mathbf{Z}^{<|\alpha|}=\prod_{\alpha \in S} \mathbf{Z}_{|\alpha|} \rightarrow \mathbf{Z} .
$$

So we see that $\operatorname{Hom}\left(\prod_{\alpha \in S} \mathbf{Z}_{|\alpha|}, \mathbf{Z}\right) \neq 0$. From a theorem of J. Loš and $|\alpha|<\boldsymbol{\aleph}_{m}$ we have $\operatorname{Hom}\left(\mathbf{Z}_{|\alpha|}, \mathbf{Z}\right)=0$ for all $\alpha \in S$; compare L. Fuchs [Fu1, Vol. II, p. 161, Theorem 94.4(ii)]. In terms of the radical $R_{\mathrm{Z}}$ we have shown

$$
R_{\mathbf{z}} \mathbf{Z}_{|\alpha|}=\mathbf{Z}_{|\alpha|} \text { for all } \alpha \in S
$$

and

$$
R_{\mathbf{z}} \prod_{\alpha \in S} \mathbf{Z}_{|\alpha|} \neq \prod_{\alpha \in S} \mathbf{Z}_{|\alpha|} .
$$

Since $|S|=\aleph_{m},(2.7)$ is shown.

REMARK 1. If we want to extend the $\aleph_{m}$-complete filter $\mathscr{F}$ without any effort to an $\boldsymbol{\aleph}_{m}$-complete ultrafilter $U$ of $I$, we may assume that $\boldsymbol{\aleph}_{m}$ is a strongly compact cardinal. Then this holds by the very definition; cf. T. Jech [J, p. 398]. From a result of $\mathbf{M}$. Magidor $[\mathbf{M}]$ we know that Con(ZFC $+\exists$ strongly compact cardinal) implies that $\operatorname{Con}\left(\mathrm{ZFC}+\boldsymbol{\aleph}_{m}\right.$ is strongly compact). In this case we derive consistency of $\left(\mathrm{ZFC}+R_{\mathrm{Z}}\right.$ does not commute with $\Pi$ ) from the existence of strongly compact cardinals. In (2.7) we have the stronger result following from the existence of $\boldsymbol{\aleph}_{m}$ that $R_{\mathrm{Z}}$ does not commute with products. D. Scott [Sc] has shown that there are no measurable cardinals in $V=L$. Consequently, from (2.4) we derive in $L$ (and many other models without $\boldsymbol{\aleph}_{m}$ ) that $R_{\mathbf{Z}}$ commutes with $\Pi$.

REMARK 2. A simple modification of the argument above will show even more that $R_{\mathbf{Z}} \Pi_{\alpha \in S} \mathbf{Z}_{|\alpha|}=0$, compare also $\S 3$. 
REMARK 3. Another way to look at the proof of (2.7) is as follows. Start from the universe and build its ultrapower over the normal measure $\mu$ and collapse this to $\mu$ or equivalently show that $\Pi_{\alpha \in S} \mathbf{Z}_{|\alpha|} / \mu \cong \mathbf{Z}^{I} / \mu$. Then observe that $\left(\mathbf{Z}^{I} / \mu\right)^{*}=0$.

3. Cardinal conditions. Let $R$ be a radical and $\kappa$ a cardinal, then we define

$$
R^{\kappa} A=\sum_{\substack{B \subseteq A \\|B|<\kappa}} R B
$$

for all abelian groups $A$. We use the following well known

Definition 3.1. A radical $R$ satisfies the cardinal condition if and only if there exists a cardinal $\kappa$ such that $R=R^{\kappa}$.

In [FOW2, Problem 2.4] the authors asked whether $R_{\mathrm{Z}}$ satisfies the cardinal condition. Assuming $\left(\mathrm{ZFC}+\nexists \aleph_{m}\right)$ we will show that the answer is no. In (3.2) we will present a more general result. Before we will do this we would like to illustrate the action of $R_{\mathrm{Z}}$ on abelian groups.

(a) If $\kappa<\aleph_{m}$, then $R_{\mathbf{Z}} \mathbf{Z}_{\kappa}=\mathbf{Z}_{\kappa}$.

(b) If $\kappa$ is a measurable cardinal then $R_{\mathbf{Z}} \mathbf{Z}_{\kappa}=0$.

Remark (a) was a consequence of Loš's theorem, cf. §2; Remark. Let $0 \neq x \in \mathbf{Z}^{\kappa} \backslash \mathbf{Z}^{<\kappa}$ and $\kappa$ a measurable cardinal. Then we can find a number $0 \neq z \in \mathbf{Z}$ such that $\left|z^{-1}\right|=\kappa$. Let $\mu$ be the $\sigma$-additive measure on $z^{-1}$ and $\tilde{\mu}: \mathbf{Z}^{\kappa} \rightarrow \mathbf{Z}$ the induced homomorphism, cf. (2.1). By definition of $\tilde{\mu}$ we have $\tilde{\mu}(x)=z \neq 0$. Therefore $x \in \operatorname{ker} \tilde{\mu}$ and since $\tilde{\mu}$ induces $\hat{\mu}: \mathbf{Z}^{\kappa} / \mathbf{Z}^{<\kappa} \rightarrow \mathbf{Z}$, we derive $x \notin R_{\mathbf{Z}} \mathbf{Z}_{\kappa}$. This implies (b).

The radical functor $R_{\mathrm{Z}}$ also takes intermediate values, as follows from [FOW2, Lemma 1.3].

(c) If $0 \rightarrow \mathbf{Z} \rightarrow A \rightarrow \mathbf{Z}^{\kappa} \rightarrow 0, \kappa<\boldsymbol{\aleph}_{m}$, is an extension of infinite order then $R_{\mathbf{Z}} A=\mathbf{Z}$.

In our next theorem the restrictions due to our set theoretic axioms will depend nicely balanced on the algebraic information of the given group $X$. Recall from T. Jech [J, p. 339] the notion of an inner model $0^{\#}$. There are many models which satisfy $\left(\mathrm{ZFC}+\mathrm{GCH}+\nexists 0^{\sharp}\right)$, e.g. $V=L$ implies this. However, $V=L$ is a much stronger assumption, compare $\mathrm{T}$. Jech $[\mathbf{J}, \S 13]$ and S. Shelah [S2].

THEOREM 3.2. (a) (ZFC $\left.+\mathrm{GCH}+\nexists 0^{\sharp}\right)$ If $X \neq 0$ is cotorsion-free, then $R_{X}$ does not satisfy the cardinal condition.

(b) (ZFC $\left.+\nexists \aleph_{m}\right)$ If $X \neq 0$ is strongly cotorsion-free, then $R_{X}$ does not satisfy the cardinal condition. 
Proof. In (a) we collect results from [DG1], [DH1] and [GS]. Suppose $R_{X}=R_{X}^{\kappa}$ for some cardinal $\kappa$. Then we can choose a cardinal $\mu>$ $\max (|X|, \kappa)$ such that the axiom $\nabla_{\mu}$ holds, compare [DG1] and [GS]. Then there exists a strongly $\mu$-free group $A$ of cardinality $\mu$ with $\operatorname{Hom}(A, X)=0$ as follows from [DH1] using techniques from [DG1], cf. also [GS, §5]. From $\operatorname{Hom}(A, X)=0$ we have $R_{X} A=A$. Since $\kappa<\mu$ and $A$ is strongly $\mu$-free, all subgroups of $A$ of cardinality $\leq \kappa$ are free. If $F$ is free, obviously $R_{X} F=\bigcap_{\varphi: F \rightarrow X} \operatorname{ker} \varphi=0$. Therefore we conclude $R_{X}^{\kappa} A=0 \neq$ $A=R_{X} A$ and (a) is shown.

(b) Suppose $R_{X}=R_{X}^{\kappa}$ for some strongly cotorsion-free group $X \neq 0$ and some cardinal $\kappa$. Choose a regular cardinal $\rho>\kappa$ and let $A=X^{\rho} / X^{<\rho}$. Since $X$ is strongly cotorsion-free also $\operatorname{Hom}(A, X)=0$ by Definition 2.1. We derive $R_{X} A=A \neq 0$. If $B \subseteq A$ and $|B| \leq \kappa$, then $|B|<\rho$. From the Wald-Loš-Lemma 2.6 we have $B \subseteq X^{\rho}$. Since $R_{X} X^{\rho}=0$ and $R_{X}$ is a subfunctor of the identity, also $R_{X} B=0$. We conclude $R_{X}^{\kappa} A=0 \neq A=$ $R_{X} A$ and (b) is shown.

4. Torsion theories which are neither singly generated nor singly cogenerated. Using the strongly cotorsion-free groups investigated in $\S 2$ and $\S 3$ we answer some open problems of torsion theories. In order to do this we will first collect some well-known facts and definitions on torsion theories. This concept was introduced by several authors in the last two decades for different reasons. The most obvious motivation is that the notion of torsion subgroups should be put into a more general and transparent frame, compare J. Lambek [L], B. Stenström [St] and references given in these books.

We will use P. Hall's notation of closure operators $A$ of classes $\mathscr{X}$ of abelian groups, i.e. $A \mathscr{X}$ is a new class of abelian groups such that $\mathscr{X} \subseteq A \mathscr{X}=A(A \mathscr{X})$. The class $\mathscr{X}$ is closed with respect to $A$ if $A \mathscr{X}=\mathscr{X}$ and $\{A, B\} \mathscr{X}$ denotes the smallest class of abelian groups containing $\mathscr{X}$ which is closed with respect to $A$ and $B$. Some special operators we use are:

$S \mathscr{X}=$ all subgroups of $\mathscr{X}$-groups,

$Q \mathscr{X}=$ all epimorphic images of $\mathscr{X}$-groups,

$\oplus \mathscr{X}=$ all direct sums of $\mathscr{X}$-groups,

$\Pi_{\kappa} \mathscr{X}=$ all cartesian products of $\leq \kappa$ groups in $\mathscr{X}$,

$\Pi \mathscr{X}=$ all cartesian products of $\mathscr{X}$-groups,

$E \mathscr{X}=$ all extensions of $\mathscr{X}$-groups by $\mathscr{X}$-groups,

and the derived operators $T=\{E, Q, \oplus\}$ and $F=\{E, \Pi, S\}$. 
If $\mathscr{T}$ and $\mathscr{F}$ are classes of abelian groups, we say that $\mathscr{T} \perp \mathscr{F}$ are orthogonal if $\operatorname{Hom}(T, F)=0$ for all $T \in \mathscr{T}$ and $F \in \mathscr{F}$. Let $\mathscr{X}^{\perp}$ be the class of all groups $Y$ with $\mathscr{X} \perp Y$ and similarly let ${ }^{\perp} \mathscr{X}$ be all $Y \perp \mathscr{X}$. A pair $(\mathscr{T}, \mathscr{F})$ is a torsion theory if $\mathscr{T} \cap \mathscr{F}=\varnothing, Q \mathscr{T}=\mathscr{T}, S \mathscr{F}=\mathscr{F}$ and for any abelian group $A$ there are $T \in \mathscr{T}$ and $F \in \mathscr{F}$ such that

$$
0 \rightarrow T \rightarrow A \rightarrow F \rightarrow 0 \text {. }
$$

This is the case if and only if a pair $(\mathscr{T}, \mathscr{F})$ is maximal with respect to $\mathscr{T} \perp \mathscr{F}$. Then $\mathscr{T}=T \mathscr{T}$ is the torsion class and $\mathscr{F}=F \mathscr{F}$ is the torsion-free class of the torsion theory $(\mathscr{T}, \mathscr{F})$. If $(T \mathscr{G}, F \mathscr{C})$ is a torsion theory then $\mathscr{G}$ generates and $\mathscr{C}$ cogenerates the torsion theory, compare [St, p. 139]. We also say that $\mathscr{C}$ cogenerates $T \mathscr{G}$ and $\mathscr{G}$ generates $F \mathscr{C}$. A torsion theory is singly generated if $\mathscr{G}$ can be a set and equivalently a group. Similarly $(T \mathscr{G}, F \mathscr{C}$ ) is singly cogenerated if $\mathscr{C}$ is a set and equivalently a singleton. Hereditary torsion theories are singly generated and singly cogenerated. A theory $(\mathscr{T}, \mathscr{F})$ is hereditary if $S \mathscr{T}=\mathscr{T}$. The classical torsion theory $(T \mathbf{Q} / \mathbf{Z}, F \mathbf{Q})$ is hereditary. Here we are interested in nonhereditary torsion theories. There are torsion theories in ZFC which are not singly cogenerated, cf. [GS, Theorem 4.1]. There are also torsion theories which are not singly generated, if we assume $\left(\mathrm{ZFC}+\mathrm{GCH}+\nexists \mathrm{O}^{\#}\right)$, cf. [GS, §5]. The latter follows with the help of a result of [DH1]. Here we want to derive an even stronger result assuming only $\left(\mathrm{ZFC}+\nexists \boldsymbol{N}_{m}\right)$.

Let $\mathscr{P}$ be the class of groups $\mathbf{Z}_{\kappa}$ for all regular $\kappa<\aleph_{m}$. Then $\mathscr{C}=\mathscr{P}^{\perp}$ is the class of all strongly cotorsion-free abelian groups defined in (2.1) and $(T \mathscr{P}, \mathscr{C})$ is a torsion theory. First we want to show the

THEOREM 4.1. $\left(\mathrm{ZFC}+\nexists \aleph_{m}\right)$ The torsion class TPP is not singly generated.

Proof. Suppose that $T \mathscr{P}=T G$ is singly generated by an abelian group $G$. In particular we have $\operatorname{Hom}\left(G, \mathbf{Z}_{\kappa}\right) \neq 0$ for all regular cardinals $\kappa$. Choose any regular $\kappa>|G|$ and apply the Wald-Loš-Lemma 2.6 to show that $\mathbf{Z}$ is an epimorphic image of $G$ and therefore $\mathbf{Z} \in T \mathscr{P}$. On the other hand $\mathbf{Z} \in \mathscr{P}^{\perp}=\mathscr{C}$, which follows from the J. Loš-Theorem, cf. L. Fuchs [Fu1, Vol. II, p. 161, Theorem 94.4]. Therefore $\mathbf{Z} \in \mathscr{P}^{\perp}=\mathscr{C}$ and $\mathbf{Z} \in T \mathscr{P} \cap \mathscr{C}=\varnothing$ is a contradiction.

We can say more about the torsion theory $(T \mathscr{P}, \mathscr{C})$ after the next theorem which is the main result of this paper, cf. (4.4). This theorem will also be used to answer some open questions. 
THEOREM 4.2. If $G$ is a cotorsion-free group and $\lambda$ a strong limit cardinal of cofinality $\omega$ with $|G| \leq \lambda$. Then we can find an $\aleph_{1}$-free and slender abelian group $A$ of size $|A|=2^{\lambda}$ such that $\operatorname{Hom}(A, G)=0$.

REMARK. In [GS] S. Shelah and one of the authors proved a similar result. Here we require less about the cardinal $\lambda$ and we also derive a weaker implication which is $\operatorname{Hom}(A, G)=0$. Hence the proof becomes much simpler and this allows to pose more conditions on the group $A$. We want to point out that we consider the simplicity of the following proof and the additional properties $\aleph_{1}$-free and slender as a good bargain! The construction of the group $A$ will be similar to [DG2], but much simpler. It is trivial to find a suitable $\lambda$ for a given $G$.

Proof. Let $G$ be a given cotorsion-free abelian group and $\lambda$ a strong limit cardinal of cofinality $\omega$ with $|G| \leq \lambda$. Choose an increasing sequence $\left\{\lambda_{n}, n \in \omega\right\}$ of cardinals such that $\sup \left\{\lambda_{n}, n \in \omega\right\}=\lambda$ and $\lambda_{n+1} \geq 2^{\lambda_{n}}$. Then $B=\oplus_{\alpha \in \lambda} \alpha \mathbf{Z}$ is a free abelian group. We can find $2^{\lambda}$ such strictly increasing sequences $\left(\lambda_{n}^{\alpha}\right)_{n \in \omega}\left(\alpha \in 2^{\lambda}\right)$ converging to $\lambda$ such that any two have only finite intersection.

Let $\hat{B}$ and $\hat{G}$ be the $\mathbf{Z}$-adic completion of $B$ respectively of $G$. If $x \in \hat{B}$, then $x=\sum_{\alpha \in \lambda} \alpha x_{\alpha}$ with $x_{\alpha} \in \hat{\mathbf{Z}}$ and $[x]=\left\{\alpha \in \lambda, x_{\alpha} \neq 0\right\}$ denotes the support of $x$. Since $|G| \leq \lambda$ also

$$
|\operatorname{Hom}(\hat{B}, \hat{G})|=|\operatorname{Hom}(B, \hat{G})| \leq|\hat{G}|^{\lambda} \leq\left(\lambda^{\aleph_{0}}\right)^{\lambda}=\left(2^{\lambda}\right)^{\lambda}=2^{\lambda} \text {. }
$$

Therefore we can label all homomorphisms from $\hat{B}$ into $\hat{G}$ different from 0 by $\left\{\varphi_{\alpha}, \alpha \in 2^{\lambda}\right\}$. If $\alpha \in 2^{\lambda}$ then $\varphi_{\alpha} \neq 0$ and therefore we find $\beta_{\alpha} \in \lambda$ such that $\varphi_{\alpha}\left(\beta_{\alpha}\right) \neq 0$. Observe that $\varphi_{\alpha}$ is uniquely defined by its action on $\lambda$. Since $\sup \left\{\lambda_{n}^{\alpha}, n \in \omega\right\}=\lambda$ and $\beta_{\alpha}<\lambda$ there is $n_{0} \in \omega$ such that $\lambda_{n}^{\alpha}>\beta_{\alpha}$ for all $n \geq n_{0}$. Changing the label $n$ into $n-n_{0}$ we may assume $\lambda_{n}^{\alpha}>\beta_{\alpha}$ for all $n \in \omega$. Since $G$ is cotorsion-free we derive $\operatorname{Hom}(\hat{\mathbf{Z}}, G)=0$, cf. $\S 2$. From $\beta_{\alpha} \hat{\mathbf{Z}} \subseteq \hat{B}$ we find $\pi_{\alpha} \in \hat{\mathbf{Z}}$ such that $\varphi_{\alpha}\left(\beta_{\alpha} \pi_{\alpha}\right) \in \hat{G} \backslash G$. In order to choose generators for $A$ we let $\lambda^{\alpha}=\sum_{n \in \omega} \lambda_{n}^{\alpha} n$ ! and decompose $2^{\lambda}=\underline{A} \dot{\cup} \underline{B}$ as follows. Let $\alpha \in \underline{A}$ if $\varphi_{\alpha}\left(\lambda^{\alpha}\right) \in \hat{G} \backslash G$ and $\alpha \in \underline{B}$ if $\varphi_{\alpha}\left(\lambda^{\alpha}\right) \in G$. If $\alpha \in \underline{A}$ choose $O_{\alpha}=\lambda^{\alpha}$ and if $\alpha \in \underline{B}$ let $O_{\alpha}=\beta_{\alpha} \pi_{\alpha}+\lambda^{\alpha}$. In case $\underline{A}$ we have $\varphi_{\alpha}\left(O_{\alpha}\right) \notin G$ and in case $\underline{B}$ also $\varphi_{\alpha}\left(O_{\alpha}\right)=\varphi_{\alpha}\left(\beta_{\alpha} \pi_{\alpha}\right)+\varphi_{\alpha}\left(\lambda^{\alpha}\right) \notin G$ by our choice of $\pi_{\alpha}$. Observe that this construction does not use a transfinite induction!

We will consider the group $A=\left\langle B \cup\left\{O_{\alpha}, \alpha \in 2^{\lambda}\right\}\right\rangle_{*} \subset \hat{B}$. If $U \subseteq \hat{B}$, then $U_{*}$ is the pure closure. In this case of torsion-free groups we have $u \in U_{*}$, if $u \in \hat{B}$ and if there is a natural number $n$ such that $n u \in \mathrm{s} U$, i.e. $U_{*}$ is the preimage of the torsion subgroup of $\hat{B} / U$. 
We want to show that

(a) $\operatorname{Hom}(A, G)=0$.

Suppose that $\varphi: A \rightarrow G$ is a homomorphism different from 0 . Since $B \subseteq A$ and $\hat{G}$ is complete, there is a unique extension $\hat{\varphi}: \hat{A} \rightarrow \hat{G}$ of $\varphi$ and $\hat{A}=\hat{B}$. Since $\hat{\varphi} \neq 0$, we find $\alpha \in 2^{\lambda}$ such that $\hat{\varphi}=\varphi_{\alpha}$. We derive $\varphi\left(O_{\alpha}\right)=$ $\hat{\varphi}\left(O_{\alpha}\right)=\varphi_{\alpha}\left(O_{\alpha}\right) \in \hat{G} \backslash G$. This contradicts $O_{\alpha} \in A$ and $\varphi(A) \subseteq G$.

Now we continue in a new direction and want to show that

(b) $A$ is $\boldsymbol{\aleph}_{1}$-free [and hence is cotorsion-free].

If $\alpha \in 2^{\lambda}$ we can choose $\pi_{n}^{\alpha} \in \hat{\mathbf{Z}}$ and $z_{n}^{\alpha} \in \mathbf{Z}$ such that $\pi_{\alpha}=\pi_{n}^{\alpha} \cdot n !+$ $z_{n}^{\alpha}$. Simply observe that $\mathbf{Z}$ is dense in $\hat{\mathbf{Z}}$ with respect to the $\mathbf{Z}$-adic topology. Hence we can divide $\pi_{\alpha}$ by $n$ ! modulo $\mathbf{Z}$. If $\alpha \in \underline{A}$ and $m \in \omega$ let

$$
O_{\alpha}^{m}=\sum_{m \leq n \in \omega} \lambda_{n}^{\alpha} \frac{n !}{m !}
$$

if $\alpha \in \underline{B}$ let

$$
O_{\alpha}^{m}=\sum_{m \leq n \in \omega} \lambda_{n}^{\alpha} \frac{n !}{m !}+\beta_{\alpha} \pi_{m}^{\alpha}
$$

In particular we have

$$
(m+1) O_{\alpha}^{m+1}-O_{\alpha}^{m} \in B .
$$

The new elements $O_{\alpha}^{m}$ can be used to determine the purification of $\left\langle B \cup\left\{O_{\alpha}, \alpha \in 2^{\lambda}\right\}\right\rangle$ explicitly. We derive

$$
A=\left\langle B \cup\left\{O_{\alpha}^{m}, \alpha \in 2^{\lambda}, m \in \omega\right\}\right\rangle .
$$

Similar divisibility chains $\left\{O_{\alpha}^{m}, m \in \omega\right\}$ have been used in the $p$-group constructions, cf. [DG3]. Condition $(* *)$ follows immediately from $(*)$ and the constructed elements.

In order to show (b) we will use Pontrjagin's criterion for freeness, compare L. Fuchs [Fu2, Vol. I, p. 93]. Since subgroups of free groups are free, it remains to show that

(***) $F=\left\langle B \cup\left\{O_{\alpha}^{s}, \alpha \in E, s \in \omega\right\}\right\rangle$ is free for all finite sets $E \subseteq 2^{\lambda}$.

We find a free basis $M \cup N$ of $F$. Decompose $E$ into $K=E \cap \underline{A}$ and $L=E \cap \underline{B}$. Pick any $m \in \omega$ sufficiently large such that

(i) $\left[O_{\alpha}^{m}\right] \cap\left[O_{\gamma}^{m}\right]=\varnothing$ for all $\alpha \in K$ and $\gamma \in L$ and

(ii) $\left[O_{\alpha}^{m}\right] \cap\left[O_{\gamma}^{m}\right]=\left\{\begin{array}{ll}\varnothing & \text { if } \beta_{\alpha} \neq \beta_{\gamma} \\ \left\{\beta_{\alpha}\right\} & \text { if } \beta_{\alpha}=\beta_{\gamma}\end{array}\right.$ for all $\alpha, \gamma \in L$. 
Let

$$
N=\left\{\alpha \in \lambda, \alpha \notin \bigcup_{\gamma \in E}\left[O_{\gamma}^{m}\right]\right\} \cup\left\{\beta_{\gamma}, \gamma \in L\right\}
$$

and

$$
M=\left\{O_{\alpha}^{s}, s \geq m, \alpha \in E\right\} .
$$

First we want to show that $M \cup N$ is linearly independent.

Consider any sum $\sum_{\alpha \in N} \alpha z_{\alpha}+\sum_{\gamma \in E} \sum_{s \geq m} O_{\gamma}^{s} z_{s \gamma}=0$ for integers $z_{\alpha}$, $z_{s \gamma}$ which are 0 for almost all $\alpha, s, \gamma$. Then $\gamma \in K$ implies $\sum_{s \geq m} O_{\gamma}^{s} z_{s \gamma}=0$ and therefore $z_{s \gamma}=0$. If $\gamma \in L$ we have $\left[\Sigma_{s \geq m} O_{\gamma}^{s} z_{s \gamma}\right] \subseteq\left\{\beta_{\gamma}\right\}$. Then $z_{s \gamma}=0$ also in this case by our choice of $O_{\gamma}^{s}$. We derive $\sum_{\alpha \in N} \alpha z_{\alpha}=0$ and $z_{\alpha}=0$ holds trivially. Therefore $M \cup N$ must be linearly independent.

Finally we want to show that $M \cup N$ generates $F$. The inclusion $\langle M \cup N\rangle \subseteq F$ holds trivially by definition of $F$. From the definition of $O_{\alpha}^{m}$ and of $M$ and $N$ we see that $B \subseteq\langle M \cup N\rangle$. If $\gamma \in E$ then $O_{\gamma} \equiv$ $O_{\gamma}^{s} s ! \bmod B$ which implies $O_{\gamma}^{s} \in\langle M \cup N\rangle$ for all $s \in \omega$. We conclude $F=\langle M \cup N\rangle$ and (***) and (b) are shown.

Now we will show that

(c) $A$ is slender.

Since we want to avoid the use of too many brackets, let $a \uparrow \alpha=a(\alpha)$ be the value of $a \in A$ at $\alpha<2^{\lambda}$.

By R. Nunke's well-known characterization of slender groups, (c) is equivalent to saying that $\mathbf{Z}^{\omega} \nsubseteq A, J_{p} \nsubseteq A$ ( $J_{p}=p$-adic integers), $\mathbf{Q} \nsubseteq A$ and $A$ is torsion-free, compare L. Fuchs [Fu1, Vol. II, p. 165 Theorem 95.3]. Since $A$ is $\boldsymbol{\aleph}_{1}$-free by (b) and $J_{p}, \mathbf{Q}, \mathbf{Z} / p \mathbf{Z}$ are not $\boldsymbol{\aleph}_{1}$-free, it remains to show that $\mathbf{Z}^{\omega} \nsubseteq A$.

Suppose for contradiction that $\varphi: \mathbf{Z}^{\omega} \rightarrow A$ is a monomorphism. If $e_{n}=\left(\delta_{l n}\right)_{i \in \omega} \in \mathbf{Z}^{\omega}$, then $\mathbf{Z}^{(\omega)}=\bigoplus_{n \in \omega} e_{n} \mathbf{Z}$ and $\mathbf{Z}^{(\omega)} \cong \bigoplus_{n \in \omega} \varphi\left(e_{n}\right) \mathbf{Z}$ has infinite rank, which is used below. In order to derive the desired contradiction, we first show

$\left(c^{*}\right)$ There does not exist an increasing sequence $(r(n))_{n \in \omega}$ of natural numbers such that

$$
\left[\varphi\left(e_{r(n)}\right)\right] \backslash \bigcup_{i=1}^{n-1}\left[\varphi\left(e_{r(i)}\right)\right] \neq \varnothing
$$

for all $n \in \omega$.

Suppose that $(r(n))_{n \in \omega}$ is a sequence satisfying $\left(\mathrm{c}^{*}\right)$ and let $r(n)=n$ without loss of generality. By induction we can find another sequence $(k(n))_{n \in \omega}$ of natural numbers such that
(1) $k(1)=1$
(2) $k(n)<k(n+1)$ for all $n \in \omega$ 
(3) There is $n^{*} \in\left[\varphi\left(e_{n}\right)\right] \backslash \bigcup_{i=1}^{n-1}\left[\varphi\left(e_{i}\right)\right]$ such that

$$
\left(\varphi\left(e_{n}\right) \uparrow n^{*}\right) \cdot k(n) ! \notin k(n+1) ! \hat{\mathbf{Z}} \text {. }
$$

Let $x=\sum_{n \in \omega} e_{n} k(n)$ ! and compute

$$
\begin{aligned}
\varphi(x) \uparrow m^{*}=\sum_{n \geq m}\left(\varphi\left(e_{n}\right) \uparrow m^{*}\right) \cdot k(n) ! \equiv\left(\varphi\left(e_{m}\right) \uparrow m^{*}\right) k(m) ! \\
\quad \neq 0 \bmod k(m+1) ! \hat{\mathbf{Z}} .
\end{aligned}
$$

Hence $m^{*} \in[\varphi(x)]$ for all sequence $(k(n))_{n \in \omega}$ satisfying (1)-(3). Since $\varphi: \mathbf{Z}^{\omega} \rightarrow A$, also $\varphi(x) \in A$ and we find $0 \neq s, k \in \mathbf{Z}, \alpha \in 2^{\lambda}$ and an infinite subset $T \subseteq \omega$ such that

$$
s \varphi(x) \uparrow m^{*}=\left(O_{\alpha} \uparrow m^{*}\right) \cdot k \text { for all } m \in T .
$$

Now we choose a new sequence $\left(k^{\prime}(n)\right)_{n \in \omega}$ of natural numbers with the properties:

(1') $k^{\prime}(1)=1$

(2') $k^{\prime}(n)<k^{\prime}(n+1)$ for all $n \in \omega$

(3') $\left(\varphi\left(e_{n}\right) \uparrow n^{*}\right) k^{\prime}(n) ! \notin k^{\prime}(n+1) ! \hat{\mathbf{Z}}$ with $n^{*}$ from (3) for all $n \in \omega$ and in addition

(4') $\left(O_{\alpha} \uparrow n^{*}\right)^{2} \mid k^{\prime}(n)$ ! for all $n>1$.

This can be arranged easily by induction. Observe that $O_{\alpha}$ is determined already by our first sequence!

Using the same argument from above we obtain integers $s^{\prime}$ and $k^{\prime}$ different from 0 and an infinite subset $T^{\prime}$ of $T$ such that

$$
x^{\prime}=\sum_{n \in \omega} e_{n} k^{\prime}(n) ! \quad \text { and } s^{\prime} \varphi\left(x^{\prime}\right) \uparrow m^{*}=\left(O_{\alpha} \uparrow m^{*}\right) \cdot k^{\prime}
$$

for all $m \in T^{\prime}$.

We also derive

$$
\begin{aligned}
& s^{\prime} \varphi\left(x^{\prime}\right) \uparrow m^{*}=s^{\prime} \sum_{n \geq m}\left(\varphi\left(e_{n}\right) \uparrow m^{*}\right) \cdot k^{\prime}(n) ! \\
& \equiv\left(\varphi\left(e_{m}\right) \uparrow m^{*}\right) s^{\prime} k^{\prime}(m) ! \bmod k^{\prime}(m+1) ! \hat{\mathbf{Z}}
\end{aligned}
$$

and summarize

$$
\left(O_{\alpha} \uparrow m^{*}\right) k^{\prime} \equiv\left(\varphi\left(e_{m}\right) \uparrow m^{*}\right) s^{\prime} \cdot k^{\prime}(m) ! \bmod k^{\prime}(m+1) ! \hat{\mathbf{Z}}
$$

This equation, together with $\left(4^{\prime}\right)$, implies

$$
k^{\prime} \equiv\left(O_{\alpha} \uparrow m^{*}\right) y_{m} \bmod k^{\prime}(m+1) !\left(O_{\alpha} \uparrow m^{*}\right)^{-1} \hat{\mathbf{Z}} \quad \text { for some } y_{m} \in \hat{\mathbf{Z}}
$$

But because of $\left(2^{\prime}\right)$ and $\left(4^{\prime}\right)$ we get that

$$
\left(O_{\alpha} \uparrow m^{*}\right) \text { divides } k^{\prime}(m+1) !\left(O_{\alpha} \uparrow m^{*}\right)^{-1} \text {. }
$$

Hence $\left(O_{\alpha} \uparrow m^{*}\right)$ divides $k^{\prime}$ for all $m \in T^{\prime}$. 
Since $T^{\prime}$ is infinite we derive $k^{\prime}=0$ from the definition of $O_{\alpha}$, which is a contradiction and $\left(\mathrm{c}^{*}\right)$ is shown.

Now we continue our proof of (c) investigating the given monomorphism $\varphi: \mathbf{Z}^{\omega} \rightarrow A$. Since $\mathbf{Z}^{(\omega)} \cong \bigoplus_{n \in \omega} \varphi\left(e_{n}\right) \mathbf{Z}$, the set $\bigcup_{n \in \omega}\left[\varphi\left(e_{n}\right)\right]$ must be infinite. From ( $\left.\mathrm{c}^{*}\right)$ we see that $\left|\left[\varphi\left(e_{n}\right)\right]\right|$ must be infinite for almost all $n \in \omega$. Hence we may assume that $\left[\varphi\left(e_{n}\right)\right]$ is infinite for all $n \in \omega$. Since $\varphi\left(e_{n}\right) \in A$, we find a natural number $s_{n}$ and $b_{n} \in B, 0 \neq z_{\alpha n} \in \mathbf{Z}$ such that $s_{n} \varphi\left(e_{n}\right)=b_{n}+\sum_{\alpha \in \Delta_{n}} O_{\alpha} z_{\alpha n}$ for some $\Delta_{n} \subset \lambda$. If we replace $e_{n}$ by $s_{n} e_{n}$ we may also assume that $\varphi\left(e_{n}\right)=b_{n}+\sum_{\alpha \in \Delta_{n}} O_{\alpha} z_{\alpha n}$.

If $\bigcup_{n \in \omega} \Delta_{n}$ is infinite we easily produce a sequence $(r(n))_{n \in \omega}$ contradicting ( $\left.\mathrm{c}^{*}\right)$. Hence $\bigcup_{n \in \omega} \Delta_{n}$ must be finite. Obviously there exists an infinite set $T \subseteq \omega$ and some $\Delta \subseteq \bigcup_{n \in \omega} \Delta_{n}$ such that $\Delta_{n}=\Delta$ for all $n \in T$. We also assume w.l.o.g. that $T=\omega$ and summarize

$$
(+) \varphi\left(e_{n}\right)=b_{n}+\sum_{\alpha \in \Delta} O_{\alpha} z_{\alpha n} \quad \text { and } \quad z_{\alpha n} \neq 0 \quad \text { for all } n \in \omega
$$

Since $\bigoplus_{n \in \omega} \varphi\left(e_{n}\right) \mathbf{Z}$ has infinite rank and $\Delta$ is finite, $U_{n \in \omega}\left[b_{n}\right]$ must be infinite. If $\bigcup_{n \in \omega}\left[b_{n}\right] \backslash \bigcup_{\alpha \in \Delta}\left[O_{\alpha}\right]$ is infinite, we can easily find a sequence $(r(n))_{n \in \omega}$ contradicting $\left(\mathrm{c}^{*}\right)$. Hence we can assume that

$$
\bigcup_{n \in \omega}\left[b_{n}\right] \backslash \bigcup_{\alpha \in \Delta}\left[O_{\alpha}\right]
$$

is finite. Changing names we find $n^{*} \in\left(\left[b_{n}\right] \cap \bigcup_{\alpha \in \Delta}\left[O_{\alpha}\right]\right) \backslash \bigcup_{i=1}^{n-1}\left[b_{i}\right]$ for all $n \in \omega$. Since $\Delta$ is finite and $\left\{\left[O_{\alpha}\right], \alpha \in \Delta\right\}$ are almost disjoint there exists $\beta \in \Delta$ such that

$$
T=\left\{n \in \omega, n^{*} \in\left[O_{\beta}\right] \backslash \bigcup_{\alpha \in \Delta \backslash\{\beta\}}\left[O_{\alpha}\right]\right\}
$$

is infinite. We may assume $T=\omega$ without restriction and summarize that

$$
n^{*} \in\left(\left[b_{n}\right] \cap\left[O_{\beta}\right]\right) \backslash\left(\bigcup_{i=1}^{n-1}\left[b_{i}\right] \cup \bigcup_{\alpha \in \Delta \backslash\{\beta\}}\left[O_{\alpha}\right]\right) \text {. }
$$

We also choose by induction a sequence $(k(n))_{n \in \omega}$ of natural numbers such that

(I) $k(1)=1$

(II) $k(n)<k(n+1)$ for all $n \in \omega$

(III) $k(n+1) !+k(n) ! n !\left(b_{n} \uparrow n^{*}\right)$ in $\hat{\mathbf{Z}}$ for all $n \in \omega$

(IV) $\left(O_{\beta} \uparrow n^{*}\right)^{2} \mid k(n)$ ! and

(V) $k(2) !+z_{\beta 1}$. 
Now we consider $x=\sum_{n \in \omega} e_{n} k(n)$ ! and compute with (O), (II) and

$$
\begin{array}{r}
\varphi(x) \uparrow m^{*}=\sum_{n \geq m}\left(b_{n} \uparrow m^{*}\right) k(n) !+\left(O_{\beta} \uparrow m^{*}\right) \cdot \sum_{n \in \omega} z_{\beta n} \cdot k(n) ! \\
\equiv\left(b_{m} \uparrow m^{*}\right) k(m) !+\left(O_{\beta} \uparrow m^{*}\right) z \bmod k(m+1) ! \mathbf{Z} \\
\text { where } z=\sum_{n \in \mathbf{N}} z_{\beta n} k(n) !
\end{array}
$$

$(++)$

Suppose that $\varphi(x) \uparrow m^{*}=0$, then we derive from $(++)$

$$
0 \equiv\left(b_{n} \uparrow m^{*}\right) k(m) !+\left(O_{\beta} \uparrow m^{*}\right) z \bmod k(m+1) ! \hat{\mathbf{Z}}
$$

and (II) and (IV) imply $\left(O_{\beta} \uparrow m^{*}\right)^{2} \mid\left(O_{\beta} \uparrow m^{*}\right) z$. We conclude $\left(O_{\beta} \uparrow m^{*}\right) \mid z$ for all $m \in \omega$ and $z=0$ by definition of $O_{\beta}$. In particular $0=z \equiv$ $z_{\beta 1} \bmod k(2) ! \hat{\mathbf{Z}}$ which contradicts $(\mathrm{V})$. Hence we have $\varphi(x) \uparrow m^{*} \neq 0$ and $m^{*} \in[\varphi(x)] \cap\left[O_{\beta}\right]$ for almost all $m \in \omega$.

Since $\varphi(x) \in A$, we find an infinite subset $T \subseteq \omega$ and $k, s \in \mathbf{Z} \backslash\{0\}$ such that

$$
s \varphi(x) \uparrow m^{*}=\left(O_{\beta} \uparrow m^{*}\right) \cdot k \text { for all } \in T .
$$

From $(++)$ we derive

$$
s\left(b_{m} \uparrow m^{*}\right) k(m) !+s\left(O_{\beta} \uparrow m^{*}\right) z \equiv\left(O_{\beta} \uparrow m^{*}\right) k \bmod k(m+1) ! \hat{\mathbf{Z}} \text {. }
$$

Now we use (II) and (IV) and obtain $\left(O_{\beta} \uparrow m^{*}\right)^{2}|k(m) !| k(m+1)$ !. Then the last equation implies $\left(O_{\beta} \uparrow m^{*}\right) \mid(s z-k)$ and therefore

$$
s z-k=0 \text { and } k(m+1) ! \mid k(m) ! m !\left(b_{m} \uparrow m^{*}\right)
$$

for all $m \in T$ with $m \geq s$. This contradicts (III) and $A$ is slender.

At the beginning of this section we introduced the torsion theory $(T \mathscr{P}, \mathscr{C})$. Now we use $(4.2)$ to show the

COROLlaRY 4.3. The torsion theory $(T \mathscr{P}, \mathscr{C})$ is not singly cogenerated.

Proof. Assume that $(T \mathscr{P}, \mathscr{C})$ is singly cogenerated, i.e. there exists a group $H$ such that $\mathscr{C}=F\{H\}$. Since $H \in \mathscr{C}, H$ must be cotorsion-free. From Theorem 4.2 we obtain a slender group $A$ such that $\operatorname{Hom}(A, H)=0$. This implies $A \notin F\{H\}=\mathscr{C}=T \mathscr{P}^{\perp}=\mathscr{P}^{\perp}$. By definition of $\mathscr{P}$ we find a regular cardinal $\aleph_{m}>\kappa \geq \aleph_{1}$ such that $\operatorname{Hom}\left(\mathbf{Z}_{\kappa}, A\right) \neq 0$. However, $A$ is slender and a theorem of J. Loš implies that $\kappa$ is a measurable cardinal, cf. L. Fuchs [Fu1, Vol. II, p. 161, Theorem 94.4]. We conclude that $(T \mathscr{P}, \mathscr{C})$ is not singly cogenerated. 
We summarize (4.1) and (4.3).

COROllaRY 4.4. $\left(\mathrm{ZFC}+\nexists \boldsymbol{\aleph}_{m}\right)$. The torsion theory $(T \mathscr{P}, \mathscr{C})$ is neither singly generated nor singly cogenerated.

The existence of such torsion theories was unknown in $\left(\mathrm{ZFC}+\not \nexists \boldsymbol{\aleph}_{m}\right)$; (4.4) answers a problem in [FOW1].

Now 4.3 implies

COROLlaRY 4.5. (ZFC). The class $\mathscr{C}$ of all strongly cotorsion-free abelian groups cannot be obtained from an abelian group $H$ and iterated applications of the operators $S, \Pi$ and $E$, i.e. $\mathscr{C} \neq F\{H\}$ for all groups $H$.

The class of all cotorsion-free groups, in particular, is not of the form $F\{H\}$. This also answers a question in R. Göbel and B. Wald [GW2]. Using $V=L$, this was already answered in $\mathrm{M}$. Dugas and $\mathrm{G}$. Herden [DH1] and a different proof in ZFC was given in R. Göbel and S. Shelah [GS].

L. Fuchs [Fu1, p. 184] posed the following Problem 78 b. Is the class $\mathscr{S}$ of slender groups of the form $\{S, \oplus, E\}\{H\}$ for some slender groups $H$ ?

This problem was answered negatively in R. Göbel and B. Wald [GW2] using generalized growth types. Growth types which are certain subgroups of $\mathbf{Z}^{\omega}$ are introduced by E. Specker. This notation can be extended to certain subgroups of $\mathbf{Z}^{\kappa}$. In [GWW] we followed "classical proofs" on slender groups to obtain the same result more naturally. Here we obtain once more an answer to this problem of L. Fuchs as a by-product.

COROllary 4.6. There does not exist an abelian group $H$ such that $\mathscr{S}=\{S, \oplus, E\}\{H\}$ is the class of all slender groups.

Proof. Assume $\mathscr{S}=\{S, \oplus, E\}\{H\}$. Then $H \in \mathscr{S}$ is slender and, in particular, cotorsion-free. From (4.2) we obtain a slender group $A$ such that $\operatorname{Hom}(A, H)=0$. Therefore $A \notin\{S, \oplus, E\}\{H\}$ and $\mathscr{S}=$ $\{S, \oplus, E\}\{H\}$ cannot hold.

5. The Chase-radical $\nu_{1}$. In this section we will use Theorem 4.2 to derive results on the Chase-radical $\nu_{1}$, which will answer an open problem. Remember that an abelian group is $\kappa$-free for some cardinal $\kappa$ if all its subgroups of cardinality less than $\kappa$ are free. If $\mathscr{F}_{\kappa}$ is the class of all $\kappa$-free 
abelian groups, we denote by $\nu_{\kappa}$ the radical

$$
\nu_{\kappa} A=R_{\mathscr{F}_{\kappa}} A=\bigcap_{\substack{\varphi: A \rightarrow \vec{F}_{\kappa} X \\ X \in \mathscr{F}_{\kappa}}} \operatorname{ker} \varphi,
$$

compare $\S 2$. For $\kappa=\aleph_{n}$ and $n \in \omega$ we simply say $\nu_{n}=\nu_{\kappa_{n}}$ and $\nu_{1}$ is the Chase-radical. This radical was investigated thoroughly by $\mathrm{S}$. U. Chase in [C]. We denote its torsion class by $T_{1}=\left\{A, \nu_{1} A=A\right\}$, and $\left(T_{1}, \mathscr{F}_{\mathrm{s}_{1}}\right)$ is a torsion theory.

First we prove

THEOREM 5.1. The torsion theory $\left(T_{1}, \mathscr{F}_{\aleph_{1}}\right)$ is singly generated, i.e. $T_{1}=T\{H\}$ for some abelian group $H$. We can choose $H=\oplus\left[A, A^{*}=0\right.$, $\left.|A| \leq \boldsymbol{\aleph}_{0}\right]$.

REMARK. Recall that $A^{*}=\operatorname{Hom}(A, \mathbf{Z})$ and let $[A, \ldots]$ denote a class of representatives of the isomorphism classes of all groups $A$ with the property... In (5.1) this class is a set of cardinality $2^{\kappa_{0}}$.

Proof. Let $H=\oplus\left[A, A^{*}=0,|A| \leq \boldsymbol{\aleph}_{0}\right]$ and $X \in T_{1}$. We consider the largest " $H$-torsion subgroup" of $X$; this is $Y=\Sigma\{U \subseteq X, U \in T\{H\}\}$. Therefore we have $0 \rightarrow Y \rightarrow X \rightarrow V \rightarrow 0$ with $\operatorname{Hom}(H, V)=0$. Suppose $V \neq 0$. Since $X \in T_{1}$, also $V \in Q T_{1}=T_{1}$ and $V \neq 0$ cannot be $\aleph_{1}$-free. Hence we find a countable subgroup $A \subseteq V$ which is not free. From a theorem of $\mathrm{K}$. Stein we also find $0 \neq C \subseteq A$ with $C^{*}=0$, cf. L. Fuchs [Fu1, Vol. 1, p. 94, Corollary 19.3]. By definition of $H$, this group $C$ is a summand of $H$. Hence $\operatorname{Hom}(H, V) \neq 0$ is a contradiction. We conclude $V=0$ and equivalently $X=Y \in T\{H\}$, i.e. $T_{1} \subseteq T\{H\}$. Since $H \in T_{1}$, also $T\{H\} \subseteq T T_{1}=T_{1}$ and $T\{H\}=T_{1}$ is shown.

Before we continue to investigate $\nu_{1}$ we add some general and quite trivial remarks connecting radicals and torsion theories.

Proposition 5.2. Let $R$ be an idempotent radical and $T_{R}=\{A, R A=$ $A\}$ its tortion-class. Then the following conditions are equivalent

(1) $R$ satisfies the cardinal condition, i.e. $R=R^{\kappa}$ for some cardinal; compare $\S 3$.

(2) There is an abelian group $H$ such that $T_{R}=\{Q, \oplus\}\{H\}$.

Proof. (2) $\rightarrow$ (1) Assume $T_{R}=\{Q, \oplus\}\{H\}$ and let $\kappa=|H|^{+}$. It is easy to see that $T_{R}=\left\{A, S_{H} A=A\right\}$ where $S_{H} A=\sum_{\varphi: H \rightarrow A} \operatorname{Im} \varphi$. Since 
$\operatorname{Im} \varphi \in Q\{H\} \subseteq T_{R}$ and $|\operatorname{Im} \varphi| \leq|H|<\kappa$, also $R(\operatorname{Im} \varphi)=\operatorname{Im} \varphi$ and $R A$ $=R^{\kappa} A$.

(1) $\rightarrow$ (2) If $H=\oplus[A,|A|<\kappa \wedge R A=A]$, then $R H=H, H \in T_{R}$ and hence $\{Q, \oplus\}\{H\} \subseteq T_{R}$. Conversely we consider $G \in T_{R}$. We derive from (1) that $G=R G=R^{\kappa} G=\sum\{R B, B \subseteq G,|B|<\kappa\}$. If $B^{\prime}=R B$, then $R B^{\prime}=B^{\prime}$ and $\left|B^{\prime}\right|<\kappa$. Hence $B^{\prime}$ is a summand of $H$ and $G \in$ $\{Q, \oplus\}\{H\}$. Therefore $T_{R}=\{Q, \oplus\}\{H\}$ and (2) is shown.

We will also use another trivial

Observation 5.3. If $R_{X}$ is a singly generated radical, then its torsion class $T_{X}=\left\{Y, R_{X} Y=Y\right\}$ is singly cogenerated by $X$.

Proof. We have $A \in T_{X} \Leftrightarrow R_{X} A=A \Leftrightarrow \operatorname{Hom}(A, X)=0 \Leftrightarrow A \in{ }^{\perp} X$. Therefore $T_{X}={ }^{\perp} X$, i.e. $T_{X}$ is singly cogenerated.

[FOW2] ask the following question: Does the Chase-radical $\nu_{1}$ satisfy the cardinal condition?

Using (5.2) this is equivalent to ask whether we find a group $H$ such that $T_{1}=\{Q, \oplus\}\{H\}$.

From (5.1) we know a little less that $T_{1}=\{Q, \oplus, E\}\{H\}$ is singly generated (as a torsion theory). Another problem asked in [FOW1, §2] is whether $\nu_{1}=R_{X}$ is singly generated as a radical. From (5.3) we see that this implies that the torsion class $T_{1}$ is singly cogenerated. From (5.1) we obtain that $\left(T_{1}, \mathscr{F}_{\aleph_{1}}\right)$ is singly generated and singly cogenerated. Using an extension of a result of [DH2] which is shown in [GS] under the hypothesis $\left(\mathrm{ZFC}+\mathrm{GCH}+\nexists 0^{\#}\right)$ then $\left(T_{1}, \mathscr{F}_{\aleph_{1}}\right)$ should be one of the $2^{\aleph_{0}}$ torsion theories derived from prime-distributions and listed in [DH2]. Since $\left(T_{1}, \mathscr{F}_{\aleph_{1}}\right)$ does not occur, $\nu_{1}$ is not singly generated as radical in $\left(\mathrm{ZFC}+\mathrm{GCH}+\nexists 0^{\sharp}\right)$. However, we derive a stronger result in $\mathrm{ZFC}$ from (4.2).

THEOREM 5.4. $T_{1}$ is not singly cogenerated [and in particular $\nu_{1}$ is not singly generated as a radical].

Proof. Suppose that $T_{1}$ is singly cogenerated. Then we find a group $G$ such that $T_{1}={ }^{\perp} G=\{A, \operatorname{Hom}(A, G)=0\}$ and $G$ is necessarily cotorsion-free, since $G \in\left({ }^{\perp} G\right)^{\perp}=T_{1}^{\perp}=\mathscr{F}_{\aleph_{1}}$ is the class of all $\aleph_{1}$-free groups.

From Theorem 4.2 we obtain a $\aleph_{1}$-free abelian group $A \neq 0$ such that $\operatorname{Hom}(A, G)=0$. Since $A$ is $\aleph_{1}$-free we have $A \in \mathscr{F}_{\aleph_{1}}$ and since $\operatorname{Hom}(A, G)=0$ we also have $A \in T_{1}$. However $0 \neq A \in \mathscr{F}_{\mathrm{x}_{1}} \cap T_{1}$ contradicts the definition of a torsion theory $\left(T_{1}, \mathscr{F}_{\aleph_{1}}\right)$. 
We will close this section with an open problem concerning $T_{1}$. From a theorem of $\mathrm{S}$. Balcerzyk we easily see that $\Pi_{\aleph_{0}} T_{1}=T_{1}$, compare L. Fuchs [Fu1, Vol. I, p. 177, Exercise 7 and p. 176, Corollary 42.2]. However, we do not know whether $\Pi_{\kappa} T_{1}=T_{1}$ holds in general. We conjecture that already $\Pi_{\aleph_{1}} T_{1} \neq T_{1}$.

6. Radicals which are socles. In $\S 5$ we used already the notation $S_{X}$ of a socle. This is dual to the radical and in general we have

$$
S_{\mathscr{X}} A=\sum\{\operatorname{Im} \varphi, \varphi: X \rightarrow A, X \in \mathscr{X}\}
$$

for a class $\mathscr{X}$ of groups $X$. A socle $S_{\mathscr{X}}$ is singly generated if $S_{\mathscr{X}}=S_{X}$ for some group $X$. Many of the "classical" radicals share this property and are socles at the same time. Hence the question arose which radicals are also socles. [FOW2] derived the following result (Theorem 1.5):

Let $X$ be a group. Suppose for every index set $I$ that $\operatorname{Ext}\left(X^{I}, X\right)=0$. Then $R_{X}$ is a socle.

In this section we will show that this result unfortunately leads to the known classical socles only. Here we will use an older result of [GP] concerning the structure of Ext.

THEOREM 6.1. The following conditions for a group $X$ are equivalent:

(1) $\operatorname{Ext}\left(X^{\kappa}, X\right)=0$ for all cardinals $\kappa$.

(2) There are cardinals $\alpha(p), \beta(p)>0$ and $\rho$ for primes $p$ from two disjoint sets $\pi$ and $\pi^{\prime}$, respectively, such that

$$
X \cong \mathbf{Q}^{(\rho)} \oplus \bigoplus_{p \in \pi} Z\left(p^{\infty}\right)^{(\alpha(p))} \oplus \widehat{\prod_{p \in \pi^{\prime}} J_{p}^{(\beta(p))}} .
$$

Proof. (1) $\rightarrow$ (2). First we want to show that (1) implies

$\left(2^{*}\right) X$ is cotorsion.

Since bounded groups are cotorsion we may assume that $X$ is unbounded. Hence $\mathbf{Z} \subset X^{\kappa}$ and also $\mathbf{Z}^{\kappa} \subseteq X^{\kappa}$. The sequence $0 \rightarrow \mathbf{Z}^{\kappa} \rightarrow X^{\kappa}$ $\rightarrow B \rightarrow 0$ implies the exact sequence $\operatorname{Ext}\left(X^{\kappa}, X\right) \rightarrow \operatorname{Ext}\left(\mathbf{Z}^{\kappa}, X\right) \rightarrow 0$.

We apply (1) to obtain $\operatorname{Ext}\left(\mathbf{Z}^{\kappa}, X\right)=0$ for all cardinals $\kappa$.

From a result in R. Göbel and R. Prelle [GP, p. 424, Theorem] we conclude that $X$ is cotorsion and $\left(2^{*}\right)$ is shown.

If $Z_{p} \subset X$, the same argument applies to show $\operatorname{Ext}\left(Z_{p}, X\right)=0$. On the other hand we have $\operatorname{Ext}\left(Z_{p}, X\right) \cong X / p X$ and therefore $X / p X=0$. Hence $X$ is $p$-divisible. This shows that the torsion part $t X$ of $X$ must be divisible and therefore $X \cong X / t X \oplus t X$. If $t X=0$, then $X$ is torsion-free and algebraically compact by $\left(2^{*}\right)$. The structure of $X$ is then well-known 
and (2) is shown in this case. If $t X \neq 0$, then $t X=\bigoplus_{p \in \pi} Z\left(p^{\infty}\right)^{(\alpha(p))}$ for cardinals $\alpha(p)>0$ and a set $\pi \neq \varnothing$ of primes. Therefore

$$
\operatorname{Ext}\left(Z\left(p^{\infty}\right), X / t X\right)=0 \quad \text { for } p \in \pi
$$

and

$$
X / t X=\mathbf{Q}^{(\rho)} \oplus \widehat{\prod_{p \in \pi^{\prime}} J_{p}^{(\beta(p))}}
$$

for cardinals $\beta(p)>0$ and another set $\pi^{\prime}$ of primes.

Obviously $\operatorname{Ext}\left(Z\left(p^{\infty}\right), J_{q}\right)=0$ holds for all $p \in \pi$ and $q \in \pi^{\prime}$. This implies that $J_{q}$ is $p$-divisible, cf. L. Fuchs [Fu1, Vol. 1, p. 224]. We conclude $p \neq q$ and therefore $\pi \cap \pi^{\prime}=\varnothing$.

$(2) \rightarrow(1)$. Let

$$
D=\prod_{p \in \pi^{\prime}} \widehat{J_{p}^{(\beta(p))}}
$$

and observe that $D$ is torsion-free and cotorsion-free. Denote also

$$
X^{\kappa} \cong \mathbf{Q}^{\left(\rho^{\prime}\right)} \oplus \bigoplus_{p \in \pi} Z\left(p^{\infty}\right)^{\left(\alpha(p)^{\prime}\right)} \oplus D^{\kappa} .
$$

Since $X=\mathbf{Q}^{(\rho)} \oplus \oplus_{p \in \pi} Z\left(p^{\infty}\right)^{(\alpha(p))} \oplus D$ and divisible groups are injective we compute $\operatorname{Ext}\left(X^{\kappa}, X\right)=\operatorname{Ext}\left(X^{\kappa}, D\right)$. From elementary properties of cotorsion groups which can be found in L. Fuchs [Fu1, Vol. I, §54] we compute

$$
\begin{aligned}
\operatorname{Ext}\left(X^{\kappa}, X\right) & =\operatorname{Ext}\left(Q^{\left(\rho^{\prime}\right)} \oplus \bigoplus_{p \in \pi} Z\left(p^{\infty}\right)^{\left(\alpha(p)^{\prime}\right)} \oplus D^{\kappa}, D\right) \\
& =\operatorname{Ext}\left(\bigoplus_{p \in \pi} Z\left(p^{\infty}\right)^{\left(\alpha(p)^{\prime}\right)}, D\right)=\prod_{p \in \pi} \operatorname{Ext}\left(Z\left(p^{\infty}\right), D\right)^{\left.\alpha^{\prime} p\right)^{\prime}} .
\end{aligned}
$$

Since $\pi \cap \pi^{\prime}=\varnothing$, we derive $\operatorname{Ext}\left(Z\left(p^{\infty}\right), D\right)=0$ and also $\operatorname{Ext}\left(X^{\kappa}, X\right)$ $=0$.

Corollary 6.2. If $\operatorname{Ext}\left(X^{\kappa}, X\right)=0$ for all cardinals $\kappa$, then $R_{X}$ is a socle, in fact one of the following classical radicals: Let $t A$ be the torsion of $A$ and $t^{p}=\oplus_{q \neq p}(t A)_{q}$ where $(t A)_{q}$ is the $q$-component of $t A$. Let $\left(p^{\omega}: t\right) A=U$ for $U / t A=p^{\omega}(A / t A)$ be the cocomposition of $p^{\omega}$ and $t$ (in the sense of [FOW2]). If $X$ is as in (6.1)(2) and $\varepsilon_{\rho}=\mathrm{id}$ if $\rho=0$, respectively, $\varepsilon_{\rho}=t$ if $\rho \neq 0$, then we have $R_{X}=\varepsilon_{\rho} \cap \bigcap_{p \in \pi} t^{p} \cap \bigcap_{p \in \pi^{\prime}}\left(p^{\omega}: t\right)$.

REMARK. Observe that $R_{J_{p}}=\left(p^{\omega}: t\right)$. Then (6.2) follows immediately from (6.1). Hence Theorem 1.5 in [FOW2] leads only to known socles. 
COROllary 6.3. If $X$ is an abelian group such that $\operatorname{End}_{\mathbf{z}} X \varsubsetneqq \mathrm{id} \cdot \mathbf{Q}$, then $R_{X}$ is not a socle.

REMARK. Many classes (not even sets) of such groups $X$ have been constructed in a number of papers, compare [DG1] and [DG2].

Proof. Since End $_{\mathbf{z}} X \subsetneq \mathbf{Q}$, the endomorphism ring $\operatorname{End}_{\mathbf{z}} X$ is cotorsion-free by definition. Hence $X$ is also cotorsion-free as shown in [DG1, p. 323, Theorem 2.4]. From (6.1) we derive $\operatorname{Ext}\left(X^{\kappa}, X\right) \neq 0$ for some cardinal $\kappa \geq \boldsymbol{\aleph}_{0}$. The inclusion map $\left(X^{\kappa}\right)^{\left(\aleph_{0}\right)} \subseteq X^{\kappa}$ leads to the exact sequence

$$
\operatorname{Ext}\left(X^{\kappa}, X\right) \rightarrow \operatorname{Ext}\left(\left(X^{\kappa}\right)^{\left(\aleph_{0}\right)}, X\right)=\operatorname{Ext}\left(X^{\kappa}, X\right)^{\aleph_{0}} \rightarrow 0 .
$$

Since $\operatorname{Ext}\left(X^{\kappa}, X\right) \neq 0$ is divisible, $\operatorname{Ext}\left(X^{\kappa}, X\right)_{0}^{\kappa}$ contains an element of infinite order. From [FOW1, Theorem 1.4] and $\operatorname{End}_{\mathbf{z}} X \subsetneq$ id $\cdot \mathbf{Q}$ we derive that $R_{X}$ cannot be a socle.

\section{REFERENCES}

[B] R. Baer, Group theoretical properties and functions, Colloqu. Mathematicarum, 14 (1966), 280-328.

[C] S. U. Chase, On group extensions and a problem of J. H. C. Whitehead, pp. 173-193 in: "Topics in Abelian Groups", ed. J. W. Irwin and E. A. Walker, Scott, Foresman Chicago, 1963.

[DG1] M. Dugas and R. Göbel, Every cotorsion-free ring is an endomorphism ring, Proc. Lond. Math. Soc., (3) 45 (1982), 319-336.

[DG2] _ Every cotorsion-free algebra is an endomorphism algebra, Math. Z., 181 (1982), 451-470.

[DG3] _ Endomorphism algebras of torsion modules II, pp. 400-411 in "Abelian Group Theory", Proceedings of the Honolulu Conference 1982/83, Springer LNM, 1006 (1983).

[DH1] M. Dugas and G. Herden, Arbitray torsion classes and almost free abelian groups, Israel J. Math., 44 (1983), 322-334.

[DH2] Arbitrary torsion classes of abelian groups, Comm. Alg., 11 (1983), 1455-1472.

[EM] P. Eklof and A. Mekler, On constructing indecomposable groups in L, J. Algebra, 49 (1977), 96-103.

[FOW1] T. H. Fay, E. P. Oxford and G. L. Walls, Preradicals induced by homomorphisms, pp. 660-670 in Springer LNM, 1066 (1983), “Abelian Group Theory”, Proceedings, Honolulu, 1982/3.

[FOW2] _ Singly generated socles and radicals, pp. 671-684 in Springer LNM, 1066 (1983), “Abelian Group Theory”, Proceedings, Honolulu, 1982/83.

[Fr] B. Franzen, Algebraic Compactness of Filter Quotients, pp. 228-241 in Springer LNM, 874 (1981), “Abelian Group Theory”, Proceedings, Oberwolfach, 1981.

[Fu1] L. Fuchs, Infinite Abelian Groups, Academic Press New York, Vol. I (1970), Vol. II (1973). 
[Fu2] Indecomposable abelian groups of measurable cardinalities, Symposia Math., 13 (1972), 233-244.

[Ga] B. J. Gardner, When are radical classes of abelian groups closed under direct products?, Algebraic Structures and Applications, Proc. 1st West Austral. Conf. Algebra, Univ. West Austral. 1980, Lecture Notes Pure Appl. Math., 74 (1982), 87-99.

[G1] R. Göbel, On stout and slender groups, J. Algebra, 53 (1975), 39-55.

[G2] _ Endomorphism Rings of Abelian Groups, pp. 340-353 in Springer LNM, 1006 (1983), “Abelian Group Theory”, Proceedings, Honolulu 1982/83.

[G3] _ Überabzählbare abelsche Gruppen, to appear Westdeutscher Verlag Opladen 1985.

[GP] R. Göbel and R. Prelle, Solutions of two problems on cotorsion abelian groups, Arch der Math., 31 (1978), 423-431.

[GW1] R. Göbel and B. Wald, Wachstumstypen und schlanke Gruppe, Symposia Math., 23 (1979), 201-239.

[GW2] __ Lösung eines Problems von L. Fuchs, J. Algebra, 71 (1981), 219-231.

[GWW] R. Göbel, B. Wald and P. Westphal, Groups of Integer-Valued Functions, pp. 161-178 in Springer LNM 874 (1981), “Abelian Group Theory”, Proceedings, Oberwolfach 1981.

[GS] R. Göbel and S. Shelah, Semirigid classes of cotorsion-free abelian groups, to appear in J. Algebra, (1984).

[J] T. Jech, Set Theory, Academic Press, New York 1978.

[K] I, Kaplansky, Infinite Abelian Groups, Ann Arbor 1971.

[L] J. Lambeck, Torsion Theories, Additive Semantics and Rings of Quotients, Springer LNM, 177 (1971).

[L] J. Loš, Linear equations and pure subgroups, Bull. Acad. Polon. Sic., 7 (1959), 13-18.

[M] M. Magidor, How large is the first strongly compact cardinal? or A study on identity crises, Annals. Math. Logic, 10 (1976), 33-57.

[Me] A. Mekler, Proper Forcing and Abelian Groups, pp. 285-303 in Springer LNM, 1006 (1983), “Abelian Group Theory”, Proceedings, Honolulu 1982/83.

[R] G. A. Reid, Almost Free Abelian Groups, Tulane Lecture Notes 1966/67.

[Ro] D. J. S. Robinson, Finiteness conditions and generalized soluble groups, Ergebnisse der Math., 62, 63 Springer, Berlin, Heidelberg, New York 1972.

[Sc] D. Scott, Measurable cardinals and constructible sets, Bull. Acad. Polon. Sci. Sér. Sci. Math. Astron. Phys., 7 (1961), 145-149.

[S1] S. Shelah, Infinite abelian groups, Whitehead problem and some constructions, Israel J. Math., 18 (1974), 243-256.

[S2] _ Diamonds uniformizations, to appear.

[St] B. Stenström, Rings and Quotients, Grundlehren der math. Wiss., 217 Springer, Berlin, Heidelberg, New York 1975.

[W1] B. Wald, Martin axiom und die Beschreibung gewisser Homomorphismen in der Theorie der $\aleph_{1}$-freien Gruppen, Manuscripta Math., 42 (1983), 297-309.

[W2] _ On $\kappa$-products modulo $\mu$-products, pp. 362-370 in Springer LNM, 1006 (1983), “Abelian Group Theory”, Proceedings, Honolulu 1982/83.

Received November 22, 1983 and in revised form February 22, 1984. 


\title{
PACIFIC JOURNAL OF MATHEMATICS EDITORS
}

DONALD BABBITT (Managing Editor)
University of California
Los Angeles, CA 90024
CHARLEs R. DEPrIMA
California Institute of Technology
Pasadena, CA 91125
R. FINN
Stanford University
Stanford, CA 94305

DoNAld BABBitT (Managing Editor)

Hermann FlaschKa

University of Arizona

Tucson, AZ 85721

RAmesh A. Gangolli

University of Washington

Seattle, WA 98195

ROBION KIRBY

University of California

Berkeley, CA 94720

C. C. MOORE

University of California

Berkeley, CA 94720

\author{
Hugo Rossi \\ University of Utah \\ Salt Lake City, UT 84112 \\ H. SAMELSON \\ Stanford University \\ Stanford, CA 94305 \\ HAROLD STARK \\ University of California, San Diego \\ La Jolla, CA 92093
}

ASSOCIATE EDITORS

R. ARENS

E. F. BECKENBACH
(1906-1982)

B. H. NEUMANN

F. WOLF

K. Yoshida

\section{SUPPORTING INSTITUTIONS}

UNIVERSITY OF ARIZONA

UNIVERSITY OF BRITISH COLUMBIA

CALIFORNIA INSTITUTE OF TECHNOLOGY

UNIVERSITY OF CALIFORNIA

MONTANA STATE UNIVERSITY

UNIVERSITY OF NEVADA, RENO

NEW MEXICO STATE UNIVERSITY

OREGON STATE UNIVERSITY
UNIVERSITY OF OREGON

UNIVERSITY OF SOUTHERN CALIFORNIA

STANFORD UNIVERSITY

UNIVERSITY OF HAWAII

UNIVERSITY OF TOKYO

UNIVERSITY OF UTAH

WASHINGTON STATE UNIVERSITY

UNIVERSITY OF WASHINGTON 


\section{Pacific Journal of Mathematics}

\section{Vol. 118, No. $1 \quad$ March, 1985}

Dan Amir, On Jung's constant and related constants in normed linear spaces ...1 Abdul Aziz, On the location of the zeros of certain composite polynomials . . 17 Joseph Barback, On hereditarily odd-even isols and a comparability of

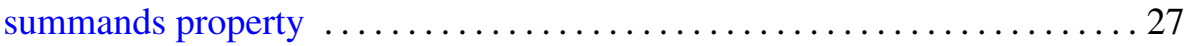

Matthew G. Brin, Klaus Johannson and Peter Scott, Totally peripheral 3-manifolds ........................................ 37

Robert F. Brown, A topological bound on the number of distinct zeros of an

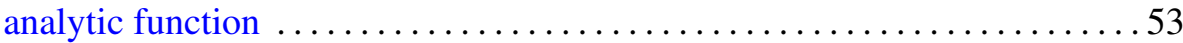

K. C. Chattopadhyay, Not every Lodato proximity is covered .......... 59

Beverly Diamond, Some properties of almost rimcompact spaces . .......63 63

Manfred Dugas and Rüdiger Göbel, On radicals and products . ......... 79

Abdelouahab El Kohen, A hyperbolic problem .................. 105

Harry Gonshor, Remarks on the Dedekind completion of a nonstandard model of the reals ................................... 117

William H. Kazez, On equivalences of branched coverings and their action

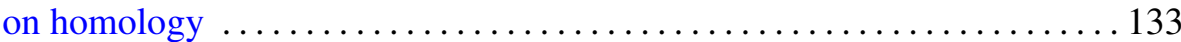

Darrell Conley Kent, On the Wallman order compactification .......... 159

Martin Andrew Magid, Lorentzian isoparametric hypersurfaces . . . . . . 165

Milan Miklavčič, Stability for semilinear parabolic equations with noninvertible linear operator

Richard Dean Neidinger and Haskell Paul Rosenthal, Norm-attainment of linear functionals on subspaces and characterizations of Tauberian operators

Johannes Vermeer, Closed subspaces of $H$-closed spaces 